\title{
Diametric Property of Spiral Wave Frequency Effect in Atrial Fibrillation
}

\author{
Donald Rubenstein ${ }^{1}$ \\ ${ }^{1}$ Prisma Health
}

September 21, 2020

\begin{abstract}
Introduction: Moving sources emitting spiral waves (SSp) such as cardiac rotors, do not exhibit frequency changes typical of classic Doppler effect (CDE) physics. Rotors exhibited fastest and slowest frequencies at either side when migrating passed electrodes, not directly in front and behind as in CDE. A new spiral wave frequency effect (SFE) equation required derivation to accurately describe and predict WF frequency changes observed near a moving SSp. Methods and Results: Rotational and spiral math were developed to derive new rotational wave frequency effect (RFE) and SFE equations in two dimensions. Wave front (WF) strikes from SR occur when clock angle equaled the line of sight (LOS) angle. WF strikes from spiral sources occurred when spiral summation angle equaled LOS angle. SFE is analyzed by varying spiral size and distance from SSp. New RFE and SFE equations predict diametric changes in frequency that occur simultaneously on either side of a passing rotor. Conclusions: WF frequency changes near a moving SSp exhibit 3 main differences compared to CDE: side-dependent frequency changes, a strong-side unpaired WF strike, and a reversal of sequence of activation. These differences, predicted by new RFE and SFE equations, constitute the unique diametric property of the rotating waves. Moving bodies that spin, or moving sources of WFs that rotate, result in perceived frequency differences that are relative to side of observation. Additionally, increasing and decreasing frequencies observed, no longer always represent an approaching and receding SR and SSp (respectively), especially when observed near the source.
\end{abstract}

\section{Methods and Results:}

Rotational and spiral math were developed to derive new rotational wave frequency effect (RFE) and SFE equations in two dimensions. Wave front (WF) strikes from $\mathrm{S}_{\mathrm{R}}$ occur when clock angle equaled the line of sight (LOS) angle. WF strikes from spiral sources occurred when spiral summation angle equaled LOS angle. SFE is analyzed by varying spiral size and distance from $\mathrm{S}_{\mathrm{Sp}}$. New RFE and SFE equations predict diametric changes in frequency that occur simultaneously on either side of a passing rotor.

\section{Conclusions:}

WF frequency changes near a moving $\mathrm{S}_{\mathrm{Sp}}$ exhibit 3 main differences compared to CDE: side-dependent frequency changes, a strong-side unpaired WF strike, and a reversal of sequence of activation. These differences, predicted by new RFE and SFE equations, constitute the unique diametric property of the rotating waves. Moving bodies that spin, or moving sources of WFs that rotate, result in perceived frequency differences that are relative to side of observation. Additionally, increasing and decreasing frequencies observed no longer always represent an approaching and receding $S_{R}$ and $S_{S p}$ (respectively), especially when observed near the source.

Keywords: Doppler Effect, spiral, rotational, rotor, atrial fibrillation, wave front, frequency effect, arrhythmia, diametric property

\section{Non-standard Abbreviations and Acronyms}


CDE - classic Doppler effect

CCW - counterclockwise

CW - clockwise

RFE - Rotational wave frequency effect

$\mathrm{RDU}$ - rotational distance unit

SFE - Spiral wave frequency effect

$\mathrm{S}_{\mathrm{C}}$ - source of centrifugally propagating wave fronts

$\mathrm{S}_{\mathrm{R}}$ - rotational wave front source, a single straight-line wave rotating about one end

$\mathrm{S}_{\mathrm{Sp}}$ - spiral wave front source, an Archimedean spiral spinning around its center

$\mathrm{T}$ - time period between wave front strikes

WF - wave front

tct - transverse common tangent between 2 circles

? - wavelength

? - line of sight angle as measured from the source's vector path being the 0 angle

C - clock angle

$\psi_{\mathrm{h}}$ - hypotenuse angle

$\mathrm{Sp}$ - spiral angle

$?_{S}-$ angular velocity

\section{Introduction}

A Doppler effect or shift describes a change in the frequency that a stationary observer senses when a source moves while emitting wave fronts at a constant frequency. Christian Doppler in 1842, observed the periodic back and forth shift in light frequency from infrared to visible red that was being emitted from binary stars in rotation (1). The higher frequency shift occurred when one star had its maximal angular velocity directly towards the Earth. The Doppler equation is derived on the assertion that periodic wave fronts (WFs) propagate centrifugally outward in expanding circles with constant propagation velocity.

\section{Three wave types, two different propagating paths}

A transverse or longitudinal WF is an oscillation of a field or particles that propagates the energy through a mass or medium without the transfer of mass itself $(2)$. Both wave types propagate outward centrifugally; the longitudinal disturbance is parallel, while the transverse disturbance is perpendicular to the path of propagation. The classic Doppler effect (CDE) and its predictive trigonometric-based equation has been confirmed in the longitudinal sound WFs and all transverse electromagnetic WFs. Fastest and slowest frequencies are observed directly in front and behind the moving source of centrifugally propagating WFs $\left(\mathrm{S}_{\mathrm{C}}\right)$, with symmetric frequency changes on either side of its motion. A recent addition to the classification of wave types is the rotational wave (3). This form of WF propagates around a central position. Physical examples of rotational WF sources $\left(\mathrm{S}_{\mathrm{R}}\right)$ ranging from micro to macro scales include circular polarized light to hurricanes and spiral galaxies. Chemical examples of spiral WFs were first realized with the BelousovZhabotinsky reaction $(4,5)$ and ultimately in electrophysiologic arrhythmias $(6)$. From here forward, it is important to further separate and distinguish rotational WF sources based upon shape difference, a rotating linear WF source $\left(\mathrm{S}_{\mathrm{R}}\right)$ and that of a spiral WF source $\left(\mathrm{S}_{\mathrm{Sp}}\right)$. Both rotate about a central core, but will have significantly different frequency change effects when comparing $S_{R}$ and $S_{S p}$ once these sources are put into motion relative to the positions of observers. 
Until now, it had been originally assumed (7), and then perpetuated (8-11), that a moving source of spiral propagating waves $\left(\mathrm{S}_{\mathrm{Sp}}\right)$ would also exhibit the classical Doppler effect (CDE). However, recent experimental evidence (12) showed repeatedly that frequency changes near a migrating cardiac rotor were too profoundly different to be explained by the CDE and its equation. Asymmetric, increasing and decreasing frequencies were recorded simultaneously on either side of the migrating rotor, not directly in front and behind as would be expected from a CDE. New frequency effect equations for rotational propagation, RFE, and spiral propagation, SFE, required derivation to be provide accurate prediction of this side and spin-dependent WF strike frequencies from either an $\mathrm{S}_{\mathrm{R}}$ or $\mathrm{S}_{\mathrm{Sp}}$. Clarity is needed at the outset; the name Doppler effect and the Doppler equations are specific to WFs that have an outward centrifugal path of propagation. Other rotational Doppler-type equations have been proposed previously for $\mathrm{S}_{\mathrm{C}}$ that only emit centrifugal propagating electromagnetic WFs (13) and are not to be confused with the frequency effect equations of WFs that have a rotational propagation path.

\section{Cardiac electrophysiologic wave fronts - sinus rhythm and rotor classification}

A normal heart beat, is the result of an electrical WF called an action potential, that propagates as a sudden depolarizing change of the cellular membrane voltage. The electrical WF propagates from pacemaker cells within the right atrium across the syncytium of cardiac cells as it is transduced to a single forceful mechanical contraction. Investigative research into this cardiac electrophysiology is vast and the reader is directed to excellent reviews $(14,15)$. The WF propagates to neighboring cells via end-to-end and side-to-side cellular intercellular gap junction connexin proteins. Although conduction of the impulse during normal sinus rhythm may propagate anisotropically (16), the action potential conduction is an aggregate of both longitudinal and transverse waves that propagate roughly on a centrifugal path outward from the sinus node.

During reentrant forms of cardiac arrhythmia, the WF propagation path is altered to follow a circuit-like path. The upstroke of the action potential propagates back around into cells recovering from their refractory period, aptly named head-meets-tail propagation. More disorganized arrhythmias, as in atrial fibrillation, a WF can pivot around a region of inexcitable tissue (permanent or functional), and break off into secondary propagating pathways. Single or multiple changing and looping paths or circuits of WF conduction can last indefinitely. The smallest of these circuits is the rotor (17). There has been mounting evidence that cardiac rotors may be a sustaining mechanism of fibrillatory activity (18-20 ). The rotor's spiral shape may be stable for 1-2 wavelengths, where one ? may range from several hundred microns up to 2-3 cm (8,21-23). Within this distance, the single WF of a cardiac rotor exhibits all 3 wave types.

\section{The SFE Deviations from the CDE}

The centrifugally outward propagation path from an $\mathrm{S}_{\mathrm{C}}$ to an observer is always along the line of sight (LOS) to that observer. Each subsequent WF is emitted as a distinctly separate oscillation of energy. Frequency is measured by the number of WFs that pass an observing site over time. In stark contrast, $\mathrm{S}_{\mathrm{Sp}}$ is a single WF in the shape of a spiral with a propagation path, especially within the first wavelength, ?, that sweeps around a center of rotation that gradually transitions to an outward path. Frequencies of WF strikes generated from an $\mathrm{S}_{\mathrm{Sp}}$ are a result of complete rotations. As $\mathrm{S}_{\mathrm{Sp}}$ moves along a vector path, the change in the period observed between WF strikes is a result not only from the change in the distance along LOS (as calculated for CDE), but also a change in the arc length of rotation of the WF. Experimentally, compass mapping of migrating rotors during human AF (12) identified profound asymmetry of WF frequencies as $\mathrm{S}_{\mathrm{Sp}}$ passed between electrodes (Figure 1A-C). One side recorded an increasing frequency, while the other side recorded a simultaneous decreasing frequency. The direction of frequency changes of were relative to the side and spin orientation of the rotor source. The side-dependent diametric frequency change was a highly consistent finding in 54 of 56 rotor transits passing the perimeter of electrodes. A 2-mm distance separated highest frequency of WF strikes to lowest frequency.

Overall, we had identified three main reproducible observations for an $\mathrm{S}_{\mathrm{S}} \mathrm{p}$ that migrated between 2 stationary electrodes: simultaneous diverging side-dependent frequency changes, an unpaired WF strike at the point of closest approach of the $\mathrm{S}_{\mathrm{Sp}}$, and a reversal of sequence of WF strikes. The path of $\mathrm{S}_{\mathrm{S} p}$ separated 2-D 
space into strong and weak-sides of recordings. The strong-side label is attached to that side which recorded increasing frequencies (during an approaching rotor) and the unpaired WF strike. These 3 events can also be found by examining other independent investigators recordings (Figure 1D, (24)). Importantly, CDE cannot predict these reproducible observations. By standard Euclidean geometry and kinematics, it will be shown below that the new RFE and SFE equations derived here predicts these 3 components and ultimately constitute a diametric property of any form of rotating sources of WFs. It will be shown that an $\mathrm{S}_{\mathrm{R}}$ or $\mathrm{S}_{\mathrm{Sp}}$ may spin at a constant angular velocity, but once that source moves relative to the position of an observer, then the frequency shifts observed is relative to the spin direction of the source and to which side of the source's motion that the observer sits. Rotational and spiral sources of WFs at levels of scale from atomic to galactic would be expected to exhibit this diametric property of RFE and SFE. Once a spinning body moves or source of rotating waves move, then frequencies changes are relative to side of motion and spin direction of the source. This newly described phenomenon of a diametric property unique to rotational WFs is easily visualized in the human heart.

\section{METHODS}

To explore most simply the fundamental symmetry differences between a moving $\mathrm{S}_{\mathrm{C}}$ and an $\mathrm{S}_{\mathrm{R}}$ or $\mathrm{S}_{\mathrm{Sp}}$, would be to compare the frequency of WF strikes at recording sites placed equidistantly on either side a vector path of the source of periodic WFs. This method will also allow the ability to derive the new RFE and SFE equations.

The WF propagation path of a hypothetical $\mathrm{S}_{\mathrm{R}}$ passes through all positions in 2-D space, ideally, during a single rotation. This sets up a new unit, a rotational distance unit (RDU), that a moving $\mathrm{S}_{\mathrm{R}}$ travelled during this one WF rotation, resulting in a WF strike at any one some specific site in this 2-D space. Each subsequent RDU provides the ability to calculate in time when the next WF strike will occur at the same site. The second step reveals that the vector path of $\mathrm{S}_{\mathrm{R}}$ splits 2-D space into 2 sides, strong and weak, that depends upon the spin direction, clockwise $(\mathrm{CW})$ or counterclockwise $(\mathrm{CCW})$. Determination of which side an observer sits relative to the vector path of the $S_{R}$ determines whether the directional change of frequency is either increasing or decreasing. This diametric property, this asymmetry, unique to rotational waves, identifies that frequency changes observed from sources that emit rotational WFs is relative to the side that one observes. Lastly, calculating the change in time periods between WF strikes from both sides and at different distances from path of motion, unveils further characteristics of this rotational relativity that differs from symmetric CDE.

\section{Rotor rotation plane and the Rotational Distance Unit}

In two dimensions, the action potential WF of a cardiac rotor has been shown to be spiral in shape. In three dimensions, the structure of the rotor is described as a scroll wave (25). The unexcited core of a scroll wave is extended perpendicularly to the rotational plane to create an unexcited filament. Computer simulations and animal experimental evidence (26-28) have shown scroll waves may have different planes of rotation. A filament that extends from the epicardial to endocardial surface is termed a transmural scroll wave. Atrial chambers are thin, only a few $\mathrm{mm}$ in thickness. Most meandering scroll waves were found to exist in the thinnest regions were transmural (26). As such, the derivation of equations is in two-dimension but could be expanded to three in later investigation.

A simplified $S_{R}$ is used as a foundational method to calculate the period between WF strikes as the $S_{R}$ moves relative to stationary observers (electrodes). The WF crest of $S_{R}$ has the shape of a straight-line segment, or ray, that pivots around a central core. The methods used here to derive the RFE equation were essential to derive the SFE equation. The new parameters developed here, methods, and graphical presentation provide the ability to solve frequency related phenomena from rotational or spiral WFs that appear in other forms of nature.

In Figure 2, an $\mathrm{S}_{\mathrm{R}}$ emits a clockwise rotational WF of constant angular velocity $\left(\omega_{\mathbf{s}}\right)$ and moves with a constant velocity $\left(\mathrm{v}_{\mathrm{s}}\right)$. A single long clock hand simulates the rotation of the $\mathrm{WF}$ crest. At greater radial distances from the center of the source clock there is a proportional increase in the tangential velocity $\left(?_{\mathrm{t}}\right.$ 
) of the WF,

$$
\overline{\omega_{\mathbf{s}}=\frac{\nu_{\mathrm{t}}}{\mathbf{r}} \cdot(1)}
$$

In biologic and physical systems, the tangential velocities of WF propagation, including action potentials, are limited by physical and biophysical restrictions (see Discussion). This figure shows how a WF crest changes its position with both source motion and spin.

The RDU discussed above (Figure $2 \mathrm{~A}$ ) is defined as the distance travelled at a constant source velocity $\left(\mathrm{v}_{\mathrm{s}}\right.$ ) as the WF makes one complete revolution around its center. One realizes that $?_{\mathrm{s}}$ and $?_{\mathrm{s}}$ are inversely proportional for an RDU. An $S_{R}$ moving twice as fast, spinning twice as fast result in the same RDU,

$$
\overline{\mathbf{R D U}=\frac{\omega_{\mathbf{s}}}{\mathbf{v}_{\mathbf{s}}}, \quad(2)}
$$

One needs to convert this to a single unit of rotation by substitution of the angular velocity with $?_{\mathrm{s}}=2 ? ?$,

$$
\overline{\mathbf{R D U}=\frac{\mathbf{2 \pi}}{\mathbf{v}_{\mathbf{s}}} \mathbf{f} \quad(3)}
$$

or

$$
\overline{\mathbf{R D U}=\frac{2 \pi}{\mathbf{v}_{\mathbf{s}} \mathbf{T}} \quad(4)}
$$

and replacing $?_{\mathbf{s}} \mathbf{T}$ with $\mathrm{D}$, the distance travelled, then the denominator simplifies to

$$
\overline{\mathbf{R D U}=\frac{\mathbf{2} \pi}{\mathbf{D}} \cdot(5)}
$$

The side dependence of the WF crest position over time is clearly evident. Tangential velocities are added to $?_{\mathrm{s}}$ on one side, while on the other side it subtracted from $?_{\mathrm{s}}$.

During the time that $S_{R}$ travelled distance $D$, the wave front crest would have struck an electrode placed in any region of $2 \mathrm{D}$ space during that single revolution. The RDU allows construct of other units, parameters, and graphical presentations that will allow the accurate computation of WF strikes, and frequency calculations at any position of electrodes (or observers). The standard definition of period $(\mathrm{T})$ is used as the time between WF strikes at a specific electrode.

In Figure 2B-D, graphical presentation of this simple rotational wave between 2 electrodes simulates the experimental results previously observed. By using this clock hand method, the clock angle (c) rotates at a constant $?_{\mathrm{s}}$. The clock angle is measured by setting the vector path as the zero angle, along a 3:00 direction. The sequence of WF strikes at $\mathrm{e}_{1}$ and $\mathrm{e}_{2}$ progressively separate in time (Figure $2 \mathrm{~B}$ and $2 \mathrm{C}$ ). WF strikes at $\mathrm{e}_{1}$ becomes earlier (arc length shortens on $\mathrm{S}_{\mathrm{R}}$ 's clock, increasing frequency) and $\mathrm{e}_{2}$ become later (arc length lengthens on $S_{R}$ 's clock, decreasing frequency). Passed the inflection point ( $\mathbf{p}$ ), the sequence of activation reverses as the WF strikes at $e_{2}$ and then $e_{1}$ progressively approach the 9:00 direction. As the $\mathrm{S}_{\mathrm{R}}$ moves between the electrodes, the direct line of sight (LOS, Figure $2 \mathrm{~B}$ and $2 \mathrm{D}$ ), angle ? to $\mathrm{e}_{1}$ and $\mathrm{e}_{2}$ changes. The LOS angle, ?, has its zero angle also aligned the 3:00 position. Therefore, acute angles are present with an approaching source, but once past the inflection point $\mathrm{p}$ (where $?=90^{\circ}$ ), angle ? becomes obtuse. It is clear that during each single rotation, a WF strike only can occur when the clock angle, $p s_{\mathbf{c}}$ is equal to the LOS angle, ?, to that specific electrode (Figure 2B-D). 
WF strike : $\psi_{\mathbf{c}}=\theta$.

The change in arc length $(\mathrm{S})$ between WF strikes is a change in the angle of the clock governed by the equation

$$
\overline{S=r \theta \cdot(7)}
$$

Importantly, had an observing electrode been placed directly in front of the moving source (at inflection point $\mathbf{p}$ ), WF strikes at that position would have occurred at 3:00 with each RDU as it approached, then at 9:00 after passing $\mathbf{p}$. Thus, no change in period, no change in frequency with approaching or receding from point $\mathbf{p}$, just an instantaneous phase shift in ? by $180^{\circ}$ or by ? radians. Thus, the vector path of the $\mathrm{S}_{\mathrm{R}}$ separates 2-D space into two regions, defined as a strong and weak-side. Observers, or electrodes that sit on the strong-side sense the increasing frequency, while those on the weak-side sense simultaneously, the diametric opposite change, a decreasing frequency.

To quantitatively understand the asymmetry and diametric property observed, a hypothetical timing experiment is presented (Figure $2 \mathrm{C}, \mathrm{D}$ ) where an $\mathrm{S}_{\mathrm{R}}$ passes between 2 equidistant electrodes $\left(\mathrm{e}_{1}\right.$ and e2), or observers, all represented as synchronized clocks. The vector path of $\mathrm{S}_{\mathrm{R}}$ is a perpendicular bisector, keeping $\mathrm{e}_{1}$ and $\mathrm{e}_{2}$ equidistant to $\mathrm{S}_{\mathrm{R}}$ along its entire vector path. Both clock angle $\mathrm{c}_{\mathbf{c}}$ and LOS angle ?, have their $0^{\circ}$ angle aligned to the vector path of $\mathrm{S}_{\mathrm{R}}$ that moves along the 3:00 line. The sweeping clock hand represents the WF crest that extends far out beyond the perimeter of the clock and moves as a single wave. The center of each clock represents the exact location of $\mathrm{S}, \mathrm{e}_{1}$, and $\mathrm{e}_{2}$. A CCW rotation would reverse the designation of $(+)$ and $(-)$ angles from the $0^{\circ}$ position.

Imaginary lines extending between 3:00 to 9:00 for each of the clocks are parallel lines. It follows then that a clock hand line that extends from the center of $\mathrm{S}_{\mathrm{R}}$ to one of the electrodes at the moment of a WF strike, that line is the line of sight (LOS). The clock times are exactly the same (congruent angles) by the theorem of corresponding angles. The clocks at each electrode are large, such that the clock perimeters hit at the inflection point $\mathrm{p}$ between the 2 electrodes. The radius of the $\mathrm{e}_{1}$ clock to its perimeter is the same for the $\mathrm{e}_{2}$ clock $\left(r_{1}=r_{2}\right)$. The vector path of the $S_{R}$ is the tangent line to both clocks at $e_{1}$ and $e_{2}$ and intersect each clock's edge at the same point ( $\mathbf{p}$ ) and represents the point of the transverse common tangent (tct) of both electrode clocks in 2D space. With each rotation of the WF crest as $S_{R}$ approaches, then recedes from point $\mathrm{p}$, the change in ?, or ?? between WF strikes corresponds directly to a proportional change in the period. Whether one adds or subtracts ?? to the previous position depends upon which side (strong or weak) the electrode sits.

\section{Determining Strong and Weak Sides: 3-point sequence rule}

A sequence-based approach (Figure 2D, right inset) is used to determine whether the observing electrode $\left(e_{1}\right)$ sits on the strong or weak-side of an approaching $S_{R}$. The $S_{R}$ vector path divides the plane into 2 sides. Two points are added to the figure. One is placed on vector path,forward of the source's current position (arrow points toe $\mathbf{e}_{\mathbf{v}}$, as a reminder). The second point is an electrode $\left(\mathrm{e}_{2}\right)$ placed on the opposite side of $\mathrm{S}_{\mathrm{R}}$ 's vector path, then the sequence is analyzed as the WF sweeps passed $\mathrm{e}_{\mathrm{v}}$. The spin direction (CW or $\mathrm{CCW})$ is determined. An electrode that records a WF strikes that precedes that at $\mathrm{e}_{\mathrm{v}}$, sits on the strongside. While an electrode that records WF strikes after the $e_{v}$, sits on the weak-side. Strong-side electrodes record decreasing time periods, $\mathrm{T}$ between $\mathrm{WF}$ strikes for an approaching $\mathrm{S}_{\mathrm{R}}$, while the weak-side electrodes record an increasing $\mathrm{T}$. One now knows to subtract ?? from the previous period (faster frequency) using a strong-side electrode position for an approaching $\mathrm{S}_{\mathrm{R}}$, or add ?? to the previous period (slower frequency) using a weak-side electrode. The 3-point sequence rule remains useful whether the spin direction is $\mathrm{CW}$ or counterclockwise $(\mathrm{CCW})$ or if the source is approaching or receding from the inflection point. Therefore, subscript labels (st) and (wk) will be used for all subsequent calculations in this paper designating which side the observing electrode sits relative to a moving rotational source. The change in time period ?T can be 
easily calculated knowing that $?_{s}$ is frequency measurement and the inverse is equal to the period of rotation of the source,

$$
\overline{\mathbf{T}_{\mathrm{s}}=\frac{\mathbf{1}}{\omega_{\mathrm{s}}} \cdot(8)}
$$

The angular change in the LOS angle ? between WF strikes results in

$$
\overline{\Delta T_{\mathrm{st}}=\frac{2 \pi-\Delta \theta_{\mathrm{st}}}{\omega_{s}}(9)}
$$

and

$$
\overline{T_{\mathrm{wk}}=\frac{2 \pi-\Delta \theta_{\mathrm{wk}}}{\omega_{s}}, \quad(10)}
$$

where ?? is a positive value at a strong-side electrode, and negative value at a weak-side electrode.

\section{Rotational math and creating family of RDU lines}

The alignment of $0^{\circ}$ angle of the clock angle $\mathbf{c}_{\mathbf{c}}$ and LOS angle ? (Figure $2 \mathrm{C}$ and D) allows a direct calculation of the change of ?, and therefore the same angular change in $p s_{\mathbf{c}}$. The change in the clock angle $p s_{\mathbf{c}}$ is a direct proportional change in the time period between WF strikes, translating accurately as frequency changes.

In the example presented in Figure 2, the center of the emitting source moves at a constant velocity, $\mathrm{v}_{\mathrm{s}}=10$ $\mathrm{cm} / \mathrm{s}$, at a constant $?_{s}=10$ rotations $/ \mathrm{s}=20 ? / \mathrm{s}$ (in radians). $\mathrm{S}_{\mathrm{R}}$ moves along the perpendicular bisector path that approaches and intersects pointp. Because $\mathrm{v}_{\mathrm{s}}$ and $?_{s}$ are both constant, the RDU can be calculated. Note that time factors out, as one simplifies the RDU such that the numerator is $2 \pi$, as in Equation 2 above,

$$
R D U=\frac{\left(\frac{20 \pi}{s}\right)}{\left(\frac{10 c m}{s}\right)}=\frac{2 \pi}{1 c m}
$$

Therefore, as the clock angle rotates 1 complete revolution, from ? to -? (2?), $\mathrm{S}_{\mathrm{R}}$ will have travelled $\mathrm{D}$ distance $(\mathrm{D}=1 \mathrm{~cm})$. The RDU can be used as the slope of the line that describes how the angle $\psi_{\mathrm{c}}$ changes (radians) as a function of $\mathrm{x}$ (the distance from $\mathrm{p}$ ). If one knows the source's clock hand position at a specific point along the vector path of $\mathrm{S}_{\mathrm{R}}$, then one could plot the line on a graph (Figures $3 \mathrm{~A}$ and $\mathrm{B}$ ). Each complete revolution 2? is plotted, showing the clock hand rotation angle from $-\pi[?] \mathrm{ps}_{\mathrm{c}}[?]+\pi$ as a function of $x$ distance travelled by $S_{R}$. Using the slope-intercept form, for $\psi_{c}=f(x)$, where a represents the $\mathrm{y}$-intercept, the clock hand angle is calculated by

$$
\psi_{c}=R D U x+a \text {. }
$$

At the end of one rotation, when $\psi_{c}$ reaches 9:00 or $-\pi$, then the subsequent rotation would restart at $+\pi$ again as the clock hand continues to rotate at a constant $?_{s}$. This next RDU line would be a parallel, having the same RDU slope from + ? to $-\pi$, but the line would be one RDU closer (y-intercept, would advance by 2 ?). This would result in subsequent y-intercepts being advance by $2 \pi$. A family or series of parallel RDU lines shows exactly the clock hand angle for any $\mathrm{S}_{\mathrm{R}}$ position along its entire vector path (Figure $4 \mathrm{~B}$ green lines) by incrementing the $\mathrm{y}$-intercept by a factor $2 \pi$ for each subsequent rotation, where $\mathrm{n}$ represents the integer of the rotation number 


$$
\psi_{c}=R D U x+(a+n 2 \pi) .
$$

\section{RESULTS}

\section{Superimposing plots of LOS angle ? and clock angle ${ }_{c}$}

Figure $3 \mathrm{~A}$ and $3 \mathrm{~B}$ provides a graphical representation of how the clock angle $\psi_{\mathrm{c}}$ (green lines) and the LOS angle ? to equidistant electrode $\mathrm{e}_{1}$ and $\mathrm{e}_{2}$ (blue and red curves) as a function of the distance $\mathrm{S}_{\mathrm{R}}$ is from the inflection point. The intersection of these plots, where $\psi_{\mathrm{c}}=$ ?, shows exactly when along the vector path that a WF strikes $e_{1}$ and $e_{2}$. The family of RDU lines have a negative slope with $\mathrm{CW}$ rotation and $\mathrm{S}_{\mathrm{R}}$ moving right to left (CCW rotation have a positive slope). Because the source's vector path is a perpendicular bisector of the line between $e_{1}$ and $e_{2}$, right triangles can be created between the electrode $e_{1}$ or $e_{2}$, point $\mathbf{p}$ , and the source location. The LOS angle? plotted as a function of distance $\mathrm{x}$ from point $\mathbf{p}$ is superimposed on this graph as arccotangent curves. The arccotangent function must be used instead of tangent since the LOS angle ? has a range 0 [?] ? [?] $\pi$ for $\mathrm{e}_{1}$, and a range of 0 [?] ? [?] $-\pi$ for $\mathrm{e}_{2}$, as the source moves passed these electrodes. The LOS angle? to $\mathrm{e}_{1}$ is given a positive magnitude (uses $+\mathrm{r}$ to $\mathrm{e}_{1}$, blue curve), while ? to $e_{2}$ is given a negative magnitude (uses $-\mathrm{r}$ to $\mathrm{e}_{2}$, red curve).

$$
\overline{\theta_{\text {st }}=\operatorname{arccot}\left(\frac{-x}{r}\right) \quad(13)}
$$

and

$$
\theta_{\mathrm{wk}}=\operatorname{arccot}\left(\frac{-x}{-r}\right)
$$

Each new rotation advances $n$ by 1 in equation 12 creating a new RDU line that intersects with the arccotangent curve. In this example, an arbitrary starting point for the first RDU, begins with the source clock hand at 9:00 (or $\pi$ radians) with the source $3 \mathrm{~cm}$ away from pointp. Using the 3 -point sequence rule above, $\mathrm{e}_{1}$ sits on the strong-side of the source's vector path and $\mathrm{e}_{2}$ sits on the weak-side. The value of $\mathrm{x}$ is made negative to reverse the axis in keeping with a right to left motion of the $S_{R}$ towards point $p$. There is symmetry of the arccot curves about the x-axis. When one plots both $\psi_{\mathrm{c}}$ and ? each as a function of $\mathrm{x}$ (Figure $4 \mathrm{~B}$ ), the points of intersection (white circles) identifies when the WF strikes at $\mathrm{e}_{1}$ and $\mathrm{e}_{2}$, where the clock angle $\psi_{\mathrm{c}}=$ ? during each RDU.

As $S_{R}$ approaches, there is one WF strike per rotation. Initially, the timing of a WF strike at $e_{1}$ immediately precedes a WF strike at $e_{2}$. The time between WF strikes at $e_{1}$ and $e_{2}$ increases with each RDU as it approaches $\mathbf{p}$ (square box, $?=\pi$ or $-\pi$ ). Once $S_{R}$ has passed $\mathbf{p}$, the WF strike at $e_{2}$ is delayed to such great extent that the WF strike at $e_{2}$ now precedes the WF strike at $e_{1}$. The WF strike at $e_{1}$ was unpaired, signaling a reversal in sequence. If one placed $\mathrm{e}_{2}$ at positions closer to inflection point (shorter $\mathrm{r}_{2}$ ), then the WF strikes would exhibit less change in time periods at distance far from $\mathbf{p}$, but quite more abrupt change with RDUs closest to $\mathrm{p}$ (black curved lines). The change becomes more sudden for closer positioned electrodes to the vector path of the rotational source.

The diametric property of rotational wave frequency effect occurs, but slightly altered when $\mathrm{S}_{\mathrm{R}}$ approaches the electrodes from an angle that is not a perpendicular bisector between electrodes (Fig 4(d-f)). The vector path is constructed to still have its closest approach to $\mathrm{e}_{1}$ be at $1 \mathrm{~cm}\left(\mathbf{r}_{\mathbf{1}}\right)$, but is tilted $30^{\circ}$ compared to $4 \mathrm{~A}$. A new radius is calculated to its point of closest approach to $e_{2}$ ( $\mathbf{r}_{2}$ with different red arccotan curve). The distance between the points of closest approach to $e_{1}$ and $e_{2}$ is length of the line segment of the transverse common tangent (tct).

Rotational Wave Frequency Effect equation 
The diametric property of rotational waves requires the identification of the strong-side and weak-side of the source's movement to determine time periods between subsequent WF strikes. Each of the WF strikes from a moving rotational WF source is directly related to subsequent WF angle change between strikes (??). Strong-side changes in the angle of the WF strike results in ?? having a positive value termed now as ?? while the weak-side having a negative value, ?? ${ }_{\mathrm{wk}}$. This allows for the derivation of the rotational wave frequency effect formula.

The change in the clock hand angle $\psi_{\mathrm{c}}$ at $\mathrm{e}_{1}$, between rotation 1 and rotation 2 is ??st. Similarly, ?? $\mathrm{wk}$ represents the clock hand angle change for the WF strikes occurring during rotation 1 and rotation 2 at $\mathrm{e}_{2}$. If one knows the constant angular velocity of source's rotational wave, then the angles ??st and ?? wk equates to the exact time period $(\mathrm{T})$ change between subsequent $\mathrm{WF}$ strikes.

The period $(\mathrm{T})$ for a rotational wave is defined as the time required to make 1 complete revolution, and is inversely proportional to the angular velocity of the source's WF,

$$
\overline{T=\frac{2 \pi}{\omega_{s}} \cdot(15)}
$$

Thus, the angular velocity is frequency (?) measurement is

$$
\omega_{s}=2 \pi f
$$

On the strong-side of the source's movement, the subsequent WF strike occurs with less of a complete rotation (less arc length) or 2?-??st . The subsequent rotation results in a new period $\mathrm{T}_{\mathrm{st}}$ that is reduced by ?? ${ }_{\mathrm{st}}$ for $\mathrm{e}_{1}$, while $\mathrm{T}_{\mathrm{wk}}$ is increased by ?? $\mathrm{wk}_{\mathrm{w}}$ or as shown above, such that

$$
T_{\text {stNew }}=\left(\frac{2 \pi-\Delta \theta_{\text {st }}}{\omega_{s}}\right) \mathrm{T} \text { and } T_{\mathrm{wkNew}}=\left(\frac{2 \pi-\Delta \theta_{\mathrm{wk}}}{\omega_{s}}\right) T
$$

for $\mathrm{e}_{1}$ and $\mathrm{e}_{2}$ respectively. One can see that subtracting ?? $\mathrm{st}$ or ?? ${ }_{\mathrm{wk}}\left(? ?_{\mathrm{wk}}\right.$ has a negative value) result in a shorter or longer period between WF strikes respectively. Note that period change with subsequent RDUs, $\mathrm{T}_{\mathrm{st}}$ and $\mathrm{T}_{\mathrm{wk}}$ for $\mathrm{e}_{1}$ and $\mathrm{e}_{2}$ respectively, are not equal and opposite in time since the WF strikes are not occurring simultaneously at $\mathrm{e}_{1}$ and $\mathrm{e}_{2}$. The source had moved closer to $\mathrm{e}_{2}$ at the time of the WF strike that is recorded there. One can convert the change in period to a change in frequency observed at each electrode since period and frequency are inversely proportional,

$$
\overline{f=\frac{\omega_{s}}{2 \pi-\Delta \theta} \cdot(18)}
$$

Importantly, ?? is either ?? $\mathrm{st}$ or ?? ${ }_{\mathrm{wk}}$, they are not equal, and they are opposite in sign. The new frequency $f^{\prime}$ 'can be compared to the original frequency $\mathrm{f}^{\circ}$, where the derivedRotational Wave Frequency Effect Equation (RFE) is now

$$
f^{\prime}=\left(\frac{2 \pi}{2 \pi-\Delta \theta_{\mathrm{St}}}\right) f^{o} \quad \text { or } \quad f^{\prime}=\left(\frac{2 \pi}{2 \pi-\Delta \theta_{\mathrm{Wk}}}\right) f^{o} .
$$

And since $?=\psi_{\mathrm{c}}$ at WF strikes, the change in the clock hand angle results in

$$
f^{\prime}=\left(\frac{2 \pi}{2 \pi-\Delta \psi_{c, S t}}\right) f^{o} \quad \text { or } \quad f^{\prime}=\left(\frac{2 \pi}{2 \pi-\Delta \psi_{c, W k}}\right) f^{o} .
$$


The change in frequency for rotational waves is markedly different compared to a classic doppler effect that has linear propagation paths and unchanging propagation velocities,

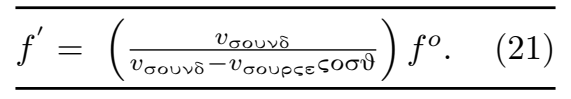

A detailed comparison is made in Fig 4 for different electrode separation from point $p$, keeping $\mathrm{e}_{1}$ and $\mathrm{e}_{2}$ equidistant to $\mathrm{S}_{\mathrm{R}}$ that revolves with a $?_{\mathrm{s}}$ at $5 \mathrm{~Hz}$. Such analysis allows further important general characteristics of the diametric property of a rotational source and can be compared to the diametric property of a spiral source $\left(\mathrm{S}_{\mathrm{Sp}}\right)$ described later.

Three main characteristics of the diametric property stand out for an approaching, then receding source of rotational WFs.

1. The strong-side position of electrodes will sense an increased frequency while simultaneously, weak-side positions will sense a decreasing frequency during the approach, with opposite changes occurring when $\mathrm{S}$ recedes from $\mathrm{p}$.

2. The strong-side will have one greater WF strike than the weak-side.

3. Reversal of sequence activation at the inflection point.

Note that the RFE shows that directly in front and behind $\mathrm{S}_{\mathrm{R}}$, (either end of the graph), the frequency of WF strikes approaches that of ? . The fastest and slowest frequencies observed are not directly in front as in $\mathrm{CDE}$, it is an asymmetric side-dependent phenomenon.

\section{THE DERIVATION OF A SPIRAL WAVE FREQUENCY EFFECT}

The cardiac rotor is not a simple rotational wave with a linear WF crest, it is spiral. The creation of the RDU in the previous rotational WF $\left(\mathrm{S}_{\mathrm{R}}\right)$ example was the essential step to derive the SFE equation. The RDU allows the accurate time calculation of a WF strike at electrodes with each single rotation from a moving source of a spiral wave $\left(\mathrm{S}_{\mathrm{Sp}}\right)$. The rotor's spiral shape is described to most nearly follow that of an Archimedean spiral (21, 29 ) with wave curvature and refractory tissue governing the ultimate period of rotation. A ray drawn from the center of the Archimedean spiral intersects each subsequent arm of the spiral at a constant distance of separation. In Figure 5, an ideal Archimedean spiral is shown. Similar to the clock hand spin, the spiral spin will also exhibit a spin and side dependence to observed frequencies. The diametric property of frequency changes exists here as well with movement of an $\mathrm{S}_{\mathrm{Sp}}$.

Determining frequency changes of WF strikes from an $\mathrm{S}_{\mathrm{Sp}}$ have additional challenges compared to that of $\mathrm{S}_{\mathrm{R}}$ of Figures 3 and 4 . First, the spiral wave, even without spinning, has a changing WF crest angle as one moves away from the emitting source's center. Second, one needs to measure the spiral wave's specific size or wavelength (?). Similar to $\mathrm{S}_{\mathrm{R}}$, the position of $\mathrm{S}_{\mathrm{Sp}}$ in 2-D described not only by the specific position of its core, but also by its rotation angle as it moves. For simplicity, it will be assumed that $\mathrm{S}_{\mathrm{Sp}}$ will maintain its spiral shape for at least 2 wavelengths out from its center, or 2 complete turns, as it moves along its vector path $\mathrm{v}_{\mathrm{s}}$. In the cardiac rotor, WF head (crest of WF) meets tail (absolute refractory period) the distance ? (between 2 arms of the spiral) should not be able to be compressed beyond this physical limit (see Discussion). The calculations and plots below of ideal spirals with ?s at similar spiral sizes, provide an accurate approximation for frequency effects as they would be recorded from electrodes near rotors during atrial fibrillation.

\section{Spiral math}

Similar to deriving RFE above, one must first develop a spiral math. The general spiral equation is

$$
r=a+\beta \psi_{\mathrm{Sp}}^{1 / c}
$$


where radius $\mathrm{r}$ and $\psi_{\mathrm{Sp}}$ identify the distance from the center and the angle of rotation to a specific point on the spiral. The value a turns the spiral, $\mathrm{b}$ is the spiral size. Figure 5A is an Archimedean spiral (c=1), and the polar wave equation is

$$
\overline{r_{\mathrm{Sp}}=a+\beta \psi_{\mathrm{Sp}} \cdot(23)}
$$

Creating a spiral clock, $\psi_{\mathrm{c}}$

To best visualize a clock hand on a spiral, the clock hand extends from the spiral's center to the point of one compete revolution (2? radians). The length of that clock hand is the spiral's size, b. This distance at that one revolution is the wavelength, ?, and is the distance between all subsequent spiral arms (Figure 5). The size $\mathrm{b}$ is derived from equation 23 by setting $\mathrm{a}=0$, and $\mathrm{r}=$ ?, and at one revolution, $\psi_{\mathrm{Sp}}=2$ ? radians,

$$
\overline{b=\frac{\lambda}{2 \pi} \cdot(24)}
$$

Substitution into equation 23 identifies a specific spiral angle $\psi_{\mathrm{Sp}}$,

$$
\overline{\psi_{\mathrm{Sp}}=\frac{2 \pi}{\lambda} r_{\mathrm{Sp}}, \quad(25)}
$$

and shows that the spiral angle $\psi_{\mathrm{Sp}}$ is inversely proportional to ? of spirals of different size. If spiral A is twice the size of spiral B, a line segment equal to spiral A's wavelength (2?) would hit the spiral B's curve at 4 ? or at two full revolutions.

From here forward, $\mathrm{S}_{\mathrm{Sp}}$ will identify a source that emits a spinning Archimedean spiral wave governed by the Equation 25. Movement of the spiral will be defined by 2 velocities, the velocity of the center of the spiral along the vector path $\left(\mathrm{v}_{\mathrm{s}}\right)$, and the angular velocity of its spin $\left(?_{\mathrm{s}}\right)$. Two angles, the clock angle $\psi$ c and the spiral angle $\psi_{\mathrm{Sp}}$, must be determined to make frequency calculations of WF strikes from a spiral wave. The clock angle $\psi_{c}$ identifies the specific position in its rotation that is referenced to a zero angle in 2-D space, while the spiral angle $\psi_{\mathrm{sp}}$ identifies the angle measured from the clock angle $\psi_{\mathrm{c}}$ to a specific position on the spiral wave. As seen in Figure 5, a spiral that spins in a clockwise direction, its spiral angle $\psi_{\text {Sp }}$ increases in the opposite, or counterclockwise direction as one measures progressively further from the spiral center along the spiral arm. Conversely, a counterclockwise spinning spiral will have a spiral angle $\psi$ $\mathrm{Sp}$ that increases in a clockwise direction.

This Archimedean spiral clock replaces the clock example of Figure 2 and 3 above. An imaginary clock hand is glued along the line segment ${ }_{0-2}$ ?, the first? (grey triangle, Figure $5 \mathrm{~B}$ and $\mathrm{C}$ ). As in the prior rotational example (Fig 2A), the center of $\mathrm{S}_{\mathrm{Sp}}$ moves along a vector path, $\mathrm{v}_{\mathrm{s}}$, toward the 3:00 position, and is used as a referenced zero angle. Therefore, the spiral's clock hand angle $\psi_{\mathrm{c}}$ is defined as the angle between the clock hand and the vector path $\mathrm{v}_{\mathrm{s}}$.

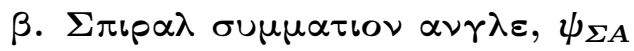

Any point on the spiral wave can now be described as a spiral summation angle $\left(\psi_{\mathrm{SA}}\right)$ of $\psi_{\mathrm{c}}$ and $\psi_{\mathrm{Sp}}$,

$$
\overline{\psi_{\mathrm{SA}}=\psi_{c}+\psi_{\mathrm{Sp}} \cdot(26)}
$$

In this example, spiral $\mathrm{S}_{\mathrm{Sp}}$ spins at a constant angular velocity, $?_{\mathrm{s}}$, that turns the clock hand angle $\psi_{\mathrm{c}}$. Any stationary observer that is placed on this 2-D plane will have its clock perfectly synchronized to that of $\mathrm{S}_{\mathrm{Sp}}$. The change in the spiral's clock angle $\psi_{\mathrm{c}}$ between any two sequential WF strikes of the spiral arm at the observer is a measure of the time period $(\mathbf{T})$ between WF strikes and can be converted to a frequency 
measurement. The T between WF strikes is measured far easier by the change in the spiral's clock angle $\psi_{\mathrm{c}}$, since the WF propagation path and velocity are always changing. If the spiral spins, but its center is stationary $\left(\mathrm{v}_{\mathrm{s}}=0\right)$, then the WF from $\mathrm{S}_{\mathrm{Sp}}$ will strike the stationary observer with a constant $\mathrm{T}$, no matter where it is positioned with each subsequent rotation. With each one revolution, the WF crest moves one ? away in distance. The frequency of WF strikes would be constant and equal to ?s. Setting the spiral in motion at constant $?_{\mathrm{s}}$, the rotation distance unit, RDU, can be utilized. The spiral will have completed one revolution after travelling $\mathrm{D}$ distance $\left(\mathrm{D}=?_{s} * \mathrm{~T}\right.$ of the RDU, Equation 5). Either the change in the spiral's clock hand angle or the change in distance the spiral had moved between each WF strike provides the change in $\mathrm{T}$ or frequency.

The $?_{s}$ vector path of $\mathrm{S}_{\mathrm{Sp}}$ in Figure 5D separates 2-D space in half. The 3-point sequence rule still applies here to designate specific sides. If 2 observers were placed one on either side of $?_{s}$, then the strong-side and weak-side nomenclature holds for an approaching $\mathrm{S}_{\mathrm{Sp}}$. WFs from the spiral move faster through the strong side and slower on the weak-side. Similar to $\mathrm{S}_{\mathrm{R}}$, at certain radial distances from the spiral center, shear zones and stall zones may exist on strong-side and weak-sides respectively. These repeating regions of shear and stall are staggered along the $\mathrm{S}_{\mathrm{Sp}}$ 's vector path separated by the distance $\mathrm{D}$ of the RDU. Examples of likely phenomena of shear and stall zones will be discussed below.

Similar to RFE, and to prove the diametric property exists for spiral waves as well, stationary electrodes $\left(\mathrm{e}_{1}\right.$ and $\mathrm{e}_{2}$ ) are placed on either side of the vector path ? equidistantly from $\mathrm{S}_{\mathrm{Sp}}$ (Figure 6). $\mathrm{S}_{\mathrm{Sp}}$ spins with a constant angular velocity $?_{\mathrm{s}}$. The activation sequence of WF strikes at electrodes $\mathrm{e}_{1}$ and $\mathrm{e}_{2}$ that straddle either side of $v_{s}$, must reverse their sequence as the spinning $\mathrm{S}_{\mathrm{Sp}}$ passes the inflection point ( $\mathrm{p}$ ) of the transverse common tangent. The LOS angle, ?, ranging from $0^{\circ}$ to $180^{\circ}$, remains the angle between the line of the forward vector path $\mathrm{v}_{\mathrm{s}}$ set at $0^{\circ}$ and the line from the center of $\mathrm{S}_{\mathrm{Sp}}$ to $\mathrm{e}_{1}$ or $\mathrm{e}_{2}$. LOS angle ? is positive if the electrode is above the vector line $v_{s}$, and negative if the electrode sits below $\mathrm{v}_{\mathrm{s}}$. Each electrode sits at the center of imaginary perfectly circular clocks created in the same 2-D plane (blue and red circles). The clock perimeters are of specific radial size, such that $v_{s}$ intersects the transverse common tangent to both clock's perimeter at point $\mathrm{p}$. Point $\mathrm{p}$ is therefore a common inflection point $(\mathrm{p})$ where the moving $S_{S p}$ changes from being an approaching $S_{S p}$ to a receding $S_{S p}$. The measured distance $h$ along the LOS angle? to either $\mathrm{e}_{1}$ or $\mathrm{e}_{2}$, is simply the hypotenuse of a right triangle that is created from $\mathrm{S}_{\mathrm{Sp}}, \mathrm{p}$, and either $\mathrm{e}_{1}$ or $\mathrm{e}_{2}$,

$$
\underline{\mathbf{h}=\sqrt{\mathbf{x}^{2}+\mathbf{r}^{\mathbf{2}}}, \quad(27)}
$$

where $\mathrm{r}$ is the radius of the imaginary clock perimeter of $\mathrm{e}_{1}$ or $\mathrm{e}_{2}$, and $\mathrm{x}$ is the distance from the $\mathrm{S}_{\mathrm{Sp}}$ to the inflection point $\mathbf{p}$. A positive value of $\mathrm{x}$ is used as the $\mathrm{S}_{\mathrm{Sp}}$ approaching $\mathrm{p}$ and negative if receding from $\mathrm{p}$ . Although the value of $\mathrm{x}$ is squared here, it's positive and negative values will need to be remembered and used later with frequency calculations. Since $e_{1}$ and $e_{2}$ are equidistant to the spiral $\mathrm{S}$ all along its path, the LOS angle?, is also equal all along its path. Any diametric changes (increasing vs decreasing) in frequency detected by the electrodes will be a result of strong and weak-side of spin direction of the spiral.

Hypotenuse used as a spiral angle, $\psi_{\mathrm{h}}$

To determine when a WF strike occurs for an Archimedean spiral at an electrode, the size of the spiral, clock angle $\psi_{\mathrm{c}}$, and position along its vector path must be known. Similar to $\mathrm{S}_{\mathrm{R}}$, the $\mathrm{S}_{\mathrm{Sp}}$ orientation angle changes all along its path, governed by $?_{\mathrm{s}}$ of the clock angle $\psi_{\mathrm{c}}$. Also, along this path of motion, the distance $\mathrm{h}$ to the electrode changes by equation 27. At each position of $\mathrm{S}_{\mathrm{Sp}}$, that new distance $\mathrm{h}$ to the electrode can be inserted into equation 25 , substituting $\mathrm{h}$ for $\mathrm{r}$ in equation 25 ,

$$
\psi_{h}=\frac{2 \pi}{\lambda_{\mathrm{Sp}}} \mathrm{h} .
$$


The calculated angle on the spiral, $\psi_{\mathrm{h}}$, and the $\mathrm{h}$ value can be oriented to the current spiral clock angle position by summing $\psi_{\mathrm{h}}+\psi_{\mathrm{c}}$, creating a new summation angle $\psi_{\mathrm{SA}}$ that replaces equation 26 ,

$\overline{\psi_{\mathrm{SA}}(x)=\psi_{c(x)}+\psi_{h(x)} \cdot(29)}$

As the $S_{S p}$ moves, the $\psi_{\mathrm{SA}}$ can be calculated all along this path of motion. Importantly, it is only when the $\psi_{\mathrm{SA}}$ is equal to the LOS angle? does a WF strike occur (Figure 6C and D). Either the change in the clock angle $\psi_{\mathrm{c}}$ between two subsequent WF strikes (first position, $\mathrm{S}_{\mathrm{Sp} 1}$ and the second position, $\mathrm{S}_{\mathrm{Sp} 2}$ ), or a measure of the distance $\mathrm{x}$ travelled by $\mathrm{S}_{\mathrm{Sp}}$ can be computed as a time period or instantaneous frequency. As seen in Figure 6C, there is a sequence reversal of WF strikes at $e_{1}$ and $e_{2}$ as $S_{S p}$ moves from position $S_{S p 1}$ (approaching $\mathrm{p}$ ) to position $\mathrm{S}_{\mathrm{Sp} 2}$ (recedes from point $\mathrm{p}$ ). A sequence reversal indicates that the frequency changes at $\mathrm{e}_{1}$ must be opposite to frequency changes at $\mathrm{e}_{2}$ (as will be shown below) and therefore the diametric property of strong and weak-sides must also exist for spiral waves.

\section{Movement of spinning spiral wave and plotting spiral summation curves}

To analyze the diametric property changes in WF strike frequency in detail, the snapshot examples of Figure 6 are put into motion as the spiral spins while travelling in a straight line. In contrast to $\mathrm{S}_{\mathrm{R}}$, WF strikes from $S_{S p}$ can be determined accurately at the intersection of the family of $\psi_{\mathrm{SA}}$ curves for each rotation (similar to family of RDU lines in Figure 3) with the arccotan curves of the LOS angle? as a function of $\mathrm{x}$ (Figure 7). Here for $\mathrm{S}_{\mathrm{Sp}}, \mathrm{WF}$ strikes occur when $\psi_{\mathrm{SA}}=$ ? for each rotation. Each clock angle $\psi_{\mathrm{c}}$ as a function of $\mathrm{x}$ is the same calculation of RDU lines as Equation 12. The plot is again limited from $-\pi$ [?] $\mathrm{ps}_{\mathrm{c}}$ $[?]+\pi$ such that at the end of one rotation, when $\psi_{\mathrm{c}}$ reaches 9:00 or $-\pi$, the y-intercept and is incremented by 2?. The hypotenuse angle $\psi_{\mathbf{h}}$, converted from the $\mathrm{h}$ distance of $\mathrm{S}_{\mathrm{Sp}}$, is added to the clock angle $\psi_{\mathrm{c}}$ for all values of $\mathbf{x}$. Substitution of the component parameters of $\psi_{\mathbf{c}}$ and $\psi_{\mathbf{h}}$ into Equation 29 yields

$$
\psi_{\mathrm{SA}}(\mathbf{x})=-\frac{2 \pi}{\mathrm{D}} \mathbf{x}+(\mathbf{a}+\mathbf{n} 2 \pi)+\frac{2 \pi}{\lambda_{\mathrm{Sp}}} \sqrt{\mathbf{x}^{2}+\mathbf{r}^{2}}
$$

which can accurately identify where on the spiral WF crest a distance $\mathrm{h}$ is measured from the spiral wave's center at any specific x-position and spin orientation. The y-intercept component essentially sets the clock time at a specific portion of an RDU. Every multiple $\mathrm{n}$ distance $\mathrm{D}$ from that position on the $\mathrm{x}$-axis will have the same $\psi_{c}$. The value a is within the range 0[?] $a[?] 2$ ?, and $\mathrm{n}$ is an integer from -[?] $<\mathrm{n}<+[?]$. For one specific $\psi_{\mathrm{SA}}$ curve, one could replace that $\mathrm{y}$-intercept with the variable $\mathrm{K}$ for a where $\mathrm{K}=\mathrm{a}+\mathrm{n} 2$ ?, and simplifies equation 30 to

$$
\overline{\psi_{\mathrm{SA}}(\mathbf{x})=-\frac{2 \pi}{\mathbf{D}} \mathbf{x}+\frac{2 \pi}{\lambda} \sqrt{\mathbf{x}^{2}+\mathbf{r}^{2}}+\mathbf{K} \cdot(31)}
$$

Thus, each spiral summation curve is made up of two components for its slope, a clock spin orientation RDU and spiral size-specific distance.

\section{Spiral wave frequency effect equation}

In the example provided in Figure 7 , the parameters of $?_{\mathrm{s}}$ and $?_{s}$ were chosen to allow easy visualization separating specific spiral positions at sequential WF strikes along vector path of $\mathrm{S}_{\mathrm{Sp}}$. Each spiral curve plotted (Figure 7A) are at the moment of subsequent WF strikes at equidistant electrodes, $\mathrm{e}_{1}$ and $\mathrm{e}_{2}$ (bold dot-dash spirals, thin dash spirals respectively). The points of intersection (white dots, Figure 7B) between the family of $\psi_{\mathrm{SA}}$ lines (black lines) with the arccotangent curves for the LOS angle ? to electrodes $\mathrm{e}_{1}$ and $\mathrm{e}_{2}$ (blue and red lines respectively) indicate exactly when a WF strike occurs $\left(\psi_{\mathrm{SA}}=\right.$ ?). The comparison of frequency relative to the side of observation can now be made (Figure $7 \mathrm{C}$ ). As the $\mathrm{S}_{\mathrm{Sp}}$ approaches both electrodes of same distance of separation, strong-side electrode $\mathrm{e}_{1}$ records an increasing frequency 
simultaneously to a weak-side decreasing frequency at $\mathrm{e}_{2}$.

These initial motion parameters were chosen specifically to provide less WF strikes than would typically occur and simplify the visualization of the diametric property. WF strikes occurred with each rotation when $\psi_{\mathrm{SA}}=$ ?. The clock angle component of $\psi_{\mathrm{SA}}$ provides the solution to frequency and must be differentiated from the clock angle of a straight arm rotational WF. The clock angle $\psi_{c}$, for WF strike calculations of the $\mathrm{RFE}$ is a direct function of?,

$$
\overline{\text { Rotational } \psi_{\mathbf{c}}(\mathbf{x})=\theta \cdot \quad(32)}
$$

To avoid confusion, by adding a superscript Sp to $\psi_{c}$, the distinction can be made where WF strikes calculation to assess the spiral wave frequency effect, is a function of $\psi_{\mathrm{SA}}-\psi_{\mathrm{h}}$,

$$
\text { Spiral } \psi_{\mathbf{c}}^{\mathrm{Sp}}(\mathbf{x})=\psi_{\mathrm{SA}(\mathbf{x})}-\psi_{\mathbf{h}(\mathbf{x})} .
$$

The RFE equation can now be modified to a spiral solution,

$$
f^{\prime}=\left(\frac{2 \pi}{2 \pi-\Delta \psi_{c-S t}^{S p}}\right) f^{o} \quad \text { or } \quad f^{\prime}=\left(\frac{2 \pi}{2 \pi-\Delta \psi_{c-W k}^{S p}}\right) f^{o} .
$$

More simply the Spiral Wave Frequency Equation (SFE) is,

$$
f^{\prime}=\left(\frac{\omega_{s}}{2 \pi-\Delta \psi_{c-S t}^{S p}}\right) \quad \text { or } \quad f^{\prime}=\left(\frac{\omega_{s}}{2 \pi-\Delta \psi_{c-W k}^{S \mathrm{~S}}}\right) \text {. }
$$

The frequency calculations from WF strikes can also be calculated most easily by using the spiral ? distancetime, $\mathrm{D}$ as a scale of frequency, and the change in $\mathrm{x}$-values between WF strikes measured against $\mathrm{D}$. Analternate SFE is,

$$
f=\frac{D}{\Delta x_{\mathrm{St}}} \omega_{s} \quad \text { or } \quad f=\frac{D}{\Delta x_{\mathrm{Wk}}} \omega_{s} .
$$

\section{f. Special case of electrode sits directly on vector path of $S_{S p}$}

Two changes occur with frequency calculations when an electrode sits directly on the $?_{\mathrm{s}}$ vector path of $\mathrm{S}_{\mathrm{Sp}}$ . First, the WF strikes would occur with LOS $?=0$, no matter the distance from inflection point $\mathrm{p}$. The subsequent WF strikes, in this special case, would occur only when $\psi_{\mathrm{SA}}=0$ radians. $\psi_{\mathrm{SA}}$ is independent of the distance from $\mathrm{p}$, and therefore not on a changing ? governed by a side-specific arccotangent curve. Second, $\mathrm{r}=0$, within the spiral size-specific distance component of the Equation 31 . The $\psi_{\mathrm{SA}}$ equation simplifies to

$$
\overline{\psi_{\mathrm{SA}}(\mathbf{x})=-\frac{2 \pi}{\mathbf{D}} \mathbf{x} \pm \frac{2 \pi}{\lambda} \mathbf{x}+\mathbf{K}, \quad(37)}
$$

where the (-) sign is used as $\mathrm{S}_{\mathrm{Sp}}$ approaches the electrode, and $(+)$ as $\mathrm{S}_{\mathrm{Sp}}$ recedes from it. The solution results in 2 families of parallel straight lines, approaching and receding. The slopes of the line or first derivative provide the ability to make a calculation of frequency, 


$$
\text { slope : } \psi^{\prime}(\mathbf{x})=\left|\frac{2 \pi}{\mathrm{D}}\right| \pm \frac{2 \pi}{\lambda} .
$$

An absolute value of the $\mathrm{D}$ component of the RDU is now used to convert the reversed $\mathrm{x}$-axis that was originally needed to measure clock angles $\psi_{\mathrm{c}}$ in Figures 3 , and 7 . The slope on the polar graph can be converted to a frequency by multiplying the slope by $\mathrm{v}_{\mathrm{s}} / 2$ ?,

$$
\mathbf{f}=\left(\left|\frac{2 \pi}{\mathrm{D}}\right| \pm \frac{2 \pi}{\lambda}\right) \frac{\mathbf{v}_{\mathbf{s}}}{2 \pi},
$$

or

$$
\overline{\mathbf{f}=\left|\frac{\mathbf{v}_{\mathbf{s}}}{\mathbf{D}}\right| \pm \frac{\mathbf{v}_{\mathbf{s}}}{\lambda} \cdot \quad(40)}
$$

This equation also provides the same result in frequency for electrodes not on the vector path $?_{\mathbf{s}}$ when $\mathrm{S}_{\mathrm{Sp}}$ is far away from the inflection point. The $\mathrm{r}$ value becomes insignificant in the calculation of the hypotenuse angle $\psi_{\mathrm{h}}$ of Equation 31. The WF strikes, intersection points on the arccotangent curves approach asymptotically the $\mathrm{x}$-axis (approach $\mathrm{y}=0$ ). It is only at relatively short distances of $\mathrm{S}_{\mathrm{Sp}}$, within the first few ? to the inflection point, that the side-dependent frequency changes, or the diametric property, would be observed. In the case of cardiac rotors with ? being up to $2-3 \mathrm{~cm}$, then the diametric property would be obvious. These frequency changes are recordings from a single site and are different than previous descriptions labelled as phase shifts comparing activation sequence at multiple different recording sites of a single revolution. The side difference is more than a mere phase shift or slip, there is an actual loss of a WF strike in number.

Both components of slope of Equation 38 have numerators of 2? which allows direct summation of angles in a polar perspective. It will be shown below that by constructing this equation in this manner, D and ? become an important ratio with regard to frequency of WF strikes when comparing approaching and receding spirals.

\section{Electrode separation, comparison of diametric property of SFE and CDE}

Cardiac rotors during atrial fibrillation move about tenfold slower than in the teaching example of Figure 7 above. Typical human cardiac rotors that have angular frequencies ranging from $4-8 \mathrm{~Hz}(30)$, and can drift at $10-20 \mathrm{~mm} / \mathrm{sec}(8)$. The diametric property is more apparent at these slower $?_{\mathbf{s}}$ rates. Computed comparisons of various electrode separation and spiral sizes are shown respectively in Figures 8 and 9 below to help identify more specific characteristics of the diametric property of the SFE. In both figures, a spiral WF source, $\mathrm{S}_{\mathrm{Sp}}$ with ? s $_{\mathrm{S}}$ at $\mathrm{Hz}$, moves $1 \mathrm{~cm} / \mathrm{sec}$ between 2 equidistant electrodes. If an electrode sat directly on the $\mathrm{v}_{\mathrm{s}}$ vector path, the approaching and receding WF strike frequency would be at a constant $5.32 \mathrm{~Hz}$ $(\mathrm{T}=188 \mathrm{msec})$ and $4.68 \mathrm{~Hz}(\mathrm{~T}=213 \mathrm{msec}$ ), respectively (using Equation 40). However, just $2 \mathrm{~mm}$ off to the side of the vector path (Figure 8, dark blue and dark red curves), the diametric property of the SFE shows that the strong-side has a peak WF frequency of $5.78 \mathrm{~Hz}(\mathrm{~T}=173 \mathrm{msec})$ while simultaneously, the weak-side has a nadir frequency of $4.24 \mathrm{~Hz}(\mathrm{~T}=238 \mathrm{msec})$. Electrodes positioned closest to the side of the vector path of $\mathrm{S}_{\mathrm{Sp}}$ have the greatest magnitude changes in frequency compared to electrodes placed further to the sides, or directly in front or back. Contrast this for a moving source, $\mathrm{S}_{\mathrm{c}}$, that emitting periodic centrifugal propagating WFs. Electrodes positioned equidistantly on either side of $?_{\mathbf{s}}$, would experience WF strikes simultaneously and the change in frequency would be governed by the classical Doppler Equation 21 as seen in Figure 8B. Peak and nadir frequency are seen directly in front or behind, not to the sides as that observed from an $\mathrm{S}_{\mathrm{Sp}}$.

\section{h. Spiral wavelength effect on diametric property of SFE, ?/D ratio, and surfing}

The diametric property of the SFE is more apparent with closer equidistant electrodes to $\mathrm{S}_{\mathrm{Sp}}$. Utilizing that observation, further assessment of the diametric property was made by varying the spiral size ? as the 
$\mathrm{S}_{\mathrm{Sp}}$ moved between electrodes spaced only $2 \mathrm{~mm}$ on either side of ?s (Figure 9). All spiral sizes exhibited the diametric property with strong and weak-sides showing their greatest difference in frequencies at the inflection point. The peak frequency of the strong-side occurred earlier than the nadir frequency on weakside, resulting in a skewed diamond-shaped separation at center. The later weak-side nadir is a result of the unpaired strong-side WF signaling a sequence reversal. The largest spiral sizes (appearing more like a radial arm of an $S_{R}$ ) approached frequency changes most similar to RFE seen in Figure 4, where approaching and receding frequencies were equal at relatively long distances from $\mathrm{p}$ with a frequency of ${ }_{s}$. At the other extreme, decreasing ?, resulted in a larger spiral-size component in the slope of the summation angle, $\psi_{\mathrm{SA}}$ of Equation 31 and Equation 38, causing greater variation between the approaching and receding $\mathrm{S}_{\mathrm{Sp}}$. In the special situation where ? $=\mathrm{D}$, the approaching frequency is twice that of $?_{s}$, and the receding frequency is zero. At this surfing ratio, there are no WF strikes because the WF crests continuously straddles the electrodes. At smaller ratio, where $? / \mathrm{D}<1$, then $\mathrm{WF}$ sequence does not reverse when $\mathrm{S}_{\mathrm{Sp}}$ recedes from $\mathrm{p}$ (not shown).

\section{DISCUSSION}

WFs emitted from rotational and spiral sources do not follow classic Doppler effect phenomena. In a previous investigation of rotors migrating passed a perimeter of electrodes in human AF (12), three new events were observed repeatedly that were unable to be predicted by the CDE equation. This is corroborated by same events that can be found when analyzing recordings of independent investigators (Figure 1D, (24)). These events, diametric side-dependent frequency changes, an unpaired strong-side WF strike, and activation reversal, constitute the diametric property of a spiral or rotational wave frequency effect. The newly derived RFE and SFE equations here, predict all 3 events of this diametric property that were seen experimentally. The Doppler effect and its equation are specific to the application of $S_{c}$, but not from a source that emits a rotating wave by an $S_{R}$ or $S_{S p}$. A first attempt to describe frequency changes from a migrating spiral wave in a Belousov - Zabotinsky reaction were "interpreted" as a phase shift of the Doppler effect (7). The technical limitations could not have identified strong and weak sides. A simple phase shift or phase slip does not accurately describe loss of a weak-side WF strike, nor simultaneous increasing and decreasing side and spin-dependent frequency changes. The importance of the asymmetry of WF strike number near a moving rotational source will be made more obvious in the examples provided below. Interestingly, the SFE results in frequency changes that asymptotically approach that calculated for a CDE after several ? of the spiral center (Figure 9).

Rotational and spiral math were developed from a new unit, the RDU, that allows accurate calculation of WF strikes with each rotation. WF strikes from a hypothetical spinning straight radial WF crest occurred only when the clock angle, $c$, equaled the LOS angle?. WF strikes from a spinning spiral WF crest occurred only when the spiral summation angle, $\mathrm{ps}_{\mathrm{SA}}$, equaled the LOS angle?. The vector line of motion of either $\mathrm{S}_{\mathrm{R}}$ or $\mathrm{S}_{\mathrm{Sp}}$ separates 2-D space into a strong-side (increasing frequencies with approaching source) and a weak-side (decreasing frequencies with approaching source). Reversing the spin direction reverses the sides. The side-dependent diametric frequency changes of both $S_{R}$ and $S_{S p}$ result from a strong-side unpaired WF strike at the inflection point when sequence reversal occurs (Figure 5 and 8). In both RFE and SFE, observation positions closer to the vector path ?s of either $S_{R}$ or $S_{S p}$ exhibited more profound frequency differences between strong and weak-sides. Observing WF strikes from a position directly on the vector path as the source passes by, results in an instantaneous phase shift with $\mathrm{S}_{\mathrm{R}}$ or an instantaneous frequency shift with $\mathrm{S}_{\mathrm{Sp}}$. Frequency changes observed on the vector path of motion for either $\mathrm{S}_{\mathrm{c}}$ or $\mathrm{S}_{\mathrm{Sp}}$ are equal. A most important conclusion from these new equations is that $2 \mathrm{~mm}$ may separate recordings of highest and lowest frequency of action potential activation, and may have just signal that a rotor had just passing between then. A bipolar electrode spanning both sides would be expected to record transient double potentials (12).

Geometrically, both $\mathrm{S}_{\mathrm{R}}$ and $\mathrm{S}_{\mathrm{Sp}}$ showed staggered potential shear and stall zones on strong and weaksides respectively which confirm likely regions of the biophysical constraints of rotor migration (11). Stall zones clearly represent regions where the electrical action potential restitution, or refractoriness, would prevent WF propagation and would be a site of a functional block (11). Shear zones, where tangential 
velocity propagation velocity hits upper biophysical limits might result in regions where the spiral shape is transformed to more centrifugal outward propagation. Additionally, the migration or sliding of the spiral core tip is limited by the electrical restitution of the atrial tissue, the critical radius of curvature, and the propagation conduction velocity restitution (11,21). Taken together, these factors that steer/curve a rotor's path, and show that a single region of atrial tissue may have multiple roles as being both active and passive contributors to the arrhythmia. Can normal atrial tissue be considered as a true source of arrhythmia if it is just the stability of the rotor's shape and its ability to recruit neighboring cells to behave abnormally transiently just as it passes through? Slight adjustment of the AF model lead to new insights into why current treatment strategies may be ineffective (32).

\section{Atrial fibrillation: The Slide-Pivot-Slide/Triggered Model, Maximizing the Slide}

The more detailed electrical recordings of migrating rotors and the identification of the diametric property of SFE allows one to slightly modify the description of atrial fibrillation. The majority of migrating rotors found in our previous study and others (12,23,24), were not sustained more than a few seconds. Our methods had selected 5 rotations or more (typically lasting at least 1 second). Even if a WF pivoted with just 1 or 2 rotations (consider naming this a swirl), great variation in the time of tissue activation, either from frequency changes, and/or phase shifts of adjacent tissue, would be manifest.

A WF crest propagates or slides between lateral boundaries, and then pivots around the end of each boundary. Continuously changing barriers from time-dependent refractory periods, constantly change the propagation path. The WF emerges beyond a barrier with a bend, a pivot, then slide between new barriers, or may even shed new $\mathrm{S}_{\mathrm{Sp}}$ (23,29,32). Focal ectopic source of APs, either from spontaneous or from triggered automaticity would add to additional drivers or functional barriers of conduction to sustain the duration of AF (33 ). New pivot points, swirls, or rotors sustain WF propagation. Just the ability to pivot to unexcited heart tissue faster than propagation of WFs from the sinus node pacemaker cells may be just as important as high dominant frequency (HDF) of transient rotors.

Observing the migration of rotors, one can conclude that any one specific atrial tissue site may change its component function of arrhythmia. Within moments, atrial cells act either as a slide, a barrier, a pivot, swirl, rotor, or a triggered site. The diametric property of SFE shows that the actual path of a rotor is a line separating highest frequency and lowest frequency. If the spin orientation was reversed, then the side of fastest and slowest frequency would also be reversed. Ablation of these moving targets of rotor migration based only upon sites of HDF might still fail. Should atrial tissue that is recruited momentarily to function abnormally be considered a true "source" of arrhythmia and ablated? Possibly, a more reasonable approach might be to shift from a moving target of normal cells behaving badly, to one that ablates regions of atrial tissue regions that have a higher ability to spawn new rotors, or regions that exhibit frequent pivots in the propagation path. New studies are being planned. Ablation may become more customized in the process to maximize the slide and minimize the pivots with a goal to limit tissue destruction.

\section{Side-Relative Frequency Perception and The Unpaired WF Strike}

When the center of an $\mathrm{S}_{\mathrm{R}}$ or $\mathrm{S}_{\mathrm{Sp}}$ is stationary but emits a rotating WF at a constant ? , all stationary observation sites record the same WF strike frequency, just at different phases of the rotation. However, once the center of an $S_{R}$ or $S_{S p}$ moves relative to stationary observers, symmetry breaks down and frequencies that are recorded are relative to the side and spin direction that one observes the $\mathrm{S}_{\mathrm{R}}$ or $\mathrm{S}_{\mathrm{Sp}}$. The vector path of motion splits 2-D space into 2 sides, a strong and weak side. Which of the two sides that an observer sits, is determined by the 3-point sequence rule described above. As an $\mathrm{S}_{\mathrm{R}}$ or $\mathrm{S}_{\mathrm{Sp}}$ moves past 2 stationary observers that are positioned on either side of the vector path, the strong-side will record one more WF strike than the weak-side. This side difference in WF strike count is independent of $?_{\mathrm{s}}$ and $?_{\mathrm{s}}$. Importantly had an observer moved together with the $\mathrm{S}_{\mathrm{R}}$ or $\mathrm{S}_{\mathrm{Sp}}$, parallel along the same vector path, keeping the same distance, then the frequency observed would be the same as ? of $\mathrm{S}_{\mathrm{R}}$ or $\mathrm{S}_{\mathrm{Sp}}$, as if neither moved.

Figure 10 provides a simple illustration where Person $\mathrm{R}$ is $\mathrm{x}$ meters from 2 closely positioned stationary observers S (strong-side) and W (weak-side). Person S and W turn their heads to keep their eyes focused 
on Person R. Person R spins at constant ${ }_{s}$ while moving at constant velocity directly between $\mathrm{S}$ and $\mathrm{W}$ to a position $\mathrm{x}$ meters away. $\mathrm{R}$ completes $1 / 2$ rotation. Person $\mathrm{R}$ faced observer $\mathrm{W}$ along its entire path and saw no turns of $\mathrm{R}$, while person $\mathrm{S}$ saw essentially one full turn. Now, in a second pass, person $\mathrm{R}$ makes $1 \frac{1}{2}$ turns, spinning again in the same direction, moving that same $2 \mathrm{x}$ meter distance. W observed 1 full turn, while S observed 2 full turns. The number of turns observed is relative to which side of the vector path that the observer sits. Independent of $?_{\mathrm{s}}$ and $?_{\mathrm{s}}, \mathrm{S}$ observes one more turn than W. Most fascinating, is that the opposite relative changes in frequency between strong and weak-sides are because of just one greater number of rotations at the inflection point between the 2 sides. The side-relative diametric frequency changes are apparent and becomes increasingly important as one investigates interactions closer to a moving $\mathrm{S}_{\mathrm{R}}$ or $\mathrm{S}_{\mathrm{Sp}}$ with its local environment or media.

This side-dependent frequency differences (diametric property of RFE or SFE) found in WFs from cardiac rotors, also apply directly to any rotating mass that moves through space relative to other objects (Figure 10C). One might ask if this diametric property affects the original Doppler conclusions? The short answer is yes, in a small way, but not in our lifetime. A hypothetical condition answers this question a bit more fully. A binary star system moves along a vector path directly between two imaginary Christian Dopplers, named $\mathrm{S}$ and $\mathrm{W}$. If the rotation plane of the binary star and the 2 Doppler observers are coplanar, then the diametric property of RFE of an $\mathrm{S}_{\mathrm{R}}$ applies. The star rotation position that results with greatest angular velocity towards each Doppler is simply when the clock angle ${ }_{c}=$ LOS angle ?. The moment that a peak frequency is observed would be identical to that of a WF strike from an $\mathrm{S}_{\mathrm{R}}$. Both Dopplers would record an oscillation in frequencies, but with marked differences in the timing of when those peaks occurred. For an approaching binary star, strong-side Doppler would record a shortening period between the peaks of frequency, as well as one greater peak count than what the weak-side Doppler records. Although the 2 men would have identified peak frequencies to occur when the tangential velocities of the star aligned with his LOS, they would have developed opposite conclusions regarding the timing of peak frequency and their theories would not have completely matched. Importantly, two other Doppler observers, D and D', that travel in the same inertial plane with the binary star, remaining the same exact distance from the binary would not observe any changes. Their plane of reference would not result in the development of strong and weak sides. Both would observe a constant frequency of the timing of the peaks, their $2 \mathrm{D}$ space would not have been split into strong and weak sides.

The proof of the existence of diametric property of rotational propagating thereby limits the definition of the Doppler effect to be a specific symmetric phenomenon only of centrifugal propagating $\mathrm{WFs}_{\mathrm{s}}$ of $\mathrm{S}_{\mathrm{C}}$. In addition, to the loss of symmetry along the sides of the path of motion of $\mathrm{S}_{\mathrm{R}}$ or $\mathrm{S}_{\mathrm{Sp}}$, is the loss of a sharp demarcation at the inflection point of perceived blue-shifted frequency (or higher frequency observed than emitted) and perceived red-shifted frequency (or lower frequency observed than emitted). When an $\mathrm{S}_{\mathrm{R}}$ or $\mathrm{S}_{\mathrm{Sp}}$ moves relative to observer positions, the diametric property of RFE and SFE expands the zones of higher and lower frequencies that can be observed. Therefore, a weak-side observer can perceive slower then emitted frequencies even though $\mathrm{S}_{\mathrm{R}}$ or $\mathrm{S}_{\mathrm{Sp}}$ is approaching. The converse being true also (shaded red and blue regions, Figures 4 and 8). It would be inaccurate to assume that higher frequencies and lower frequencies observed, represent always an approaching and receding $S_{R}$ and $S_{S p}$ respectively, especially when observers are near to the source.

The asymmetric labelling of strong-side and weak-side of hurricanes (34) describing greater tangential wind speeds and storm surges (Ekman transport) may no longer be analogous or coincidental phenomena to cardiac rotors but are likely consequential of the diametric property of rotational relativity. The energy of rotating WFs subject to the diametric property of RFE and SFE may have direct influence on mass within its media to result in the spiral lattice structure of crystal formation (35) at one level of nature to the accretions of matter into the spiral stellar arms within the galaxies at another level. The creation of one's own spiral wave and surfing between its crests for a means of propulsion would require computations of specific surfing ratio of D and ? as seen in Figure 9. The diametric property of RFE and SFE appears to be the critical link between many spinning phenomena at all sizes of nature. Future investigations of the diametric property of RFE, and SFE might improve the understanding of chemical reactions, enantiomer 
formation, air turbulence, water eddies, Reynold's number, wing lift, weather models, and plotting paths of space travel.

\section{Acknowledgements}

I gratefully acknowledge the support, multiple discussions and identification of additional references by Mason Rubenstein.

\section{REFERENCES}

1. Doppler C. About the coloured light of binary stars and some other stars in the heavens. Proceedings of the Royal Bohemian Society of Sciences, Prague (PartV)1842: 465-482. 2. Rosen J, Gothard LQ. Encyclopedia of Physical Science, Facts On File, New York, NY: An Imprint of Infobase Publishing; 2010:678-685pp. 3. Ceperley PH. Rotating waves. Am. J. Phys.1992;60:938-942. 4. Belousov BP. "A periodic reaction and its mechanism" In: Field R, Burger M, eds. Oscillations and Travelling Waves in Chemical Systems, NY: Wiley; 1985:605-613pp. 5. Zhabotinsky AM. Periodic oxidation of malonic acid in solution (a study of the Belousov reaction kinetics). Biofizika.1964;9:306-311. 6. Allessie MA, Bonke FIM, Schopman FGJ. Circus movement in rabbit atrial muscle as a mechanism of tachycardia: II. The role of nonuniform recovery of excitability. Circ. Res. 1976;39:168-177. 7. Perez-Muñuzuri V, Allev R, Vaslev B, Perez-Villar V, Kinsky VI. Super-spiral structures in an excitable medium. Nature 1991;353:740-742. 8. Davidenko JM, Pertsov AV, Salomonez R, Baxter W, Jalife J. Stationary and drifting spiral waves of excitation in isolated cardiac muscle. Nature 1992;355(6358): 349-351. 9. Karma A. Electrical alternans and spiral wave breakup in cardiac tissue. Chaos 1994;4(3):461-472. 10. Zlochiver S, Yamazaki M, Kalifa J, Berenfield O. Rotor meandering contributes to irregularity in electrograms during atrial fibrillation. Heart Rhythm 2008;5:846854. 11. Fenton FH, Cherry EM, Hastings HM, Evans SJ. Multiple mechanisms of spiral wave breakup in a model of cardiac electrical activity. Chaos 2002;12(3):852-892. 12. Rubenstein DS, Yin H, Azami SA. Compass mapping, double potentials, activation patterns can identify and track rotational activity sites in the left atrium of humans with persistent atrial fibrillation. J. Atr. Fibrillation 2018;11(2):2053. 13. Allen L, Barnett SM, Padgett MJ. Chapter 6. Rotational Frequency Shifts. In: Allen L, Barnett SM, Padgett MJ, eds. Optical Angular Momentum, London: Institute of Physics Publishing; 2003;245-270pp. 14. Kleber A, Rudy Y. Basic mechanisms of cardiac impulse propagation and associated arrhythmias. Physiol. Rev. 2004;84:431-488. 15. Bartos DC, Grandi E, Ripplinger CM. Ion channels in the heart. Compr. Physiol. 2015; 5(3):1423-1464. 16. Valderrábano M. Influence of anisotropic conduction properties in the propagation of the cardiac action potential. Prog. Biophys.Mol. Biol. 2007;94(1-2):144-168. 17. Gray RA, Pertsov AM, Jalife J. Spatial and temporal organization during cardiac fibrillation. Nature 1998; 392(6671):75-78. 18. Mandapati R, Skanes RA, Chen J, Berenfeld O, Jalife J. Stable microreentrant sources as a mechanism of atrial fibrillation in the isolated sheep heart. Circulation 2000;101: 194-199. 19. Jalife J, Berenfeld O, Mansour M. Mother rotors and fibrillatory conduction: a mechanism of atrial fibrillation. Cardiovasc. Res.2002;54: 204-216. 20. Quintanilla JG, Alfonso-Amazán JM, Pérez-Castellano N, Pandit SV, Jalife J, Pérez-Villacastin J, Filgueiras-Rama D. Instantaneous amplitude and frequency modulations detect the footprint of rotational activity and reveal stable driver regions as targets for persistent ablation. Circ. Research 2019; 125: 609-627. 21. Fast AG, Kléber AG. Role of wavefront curvature in propagation of cardiac impulse. Cardiovasc. Res. 1997;33:258-271. 22. Pertsov AM, Davidenko JM, Salomonsz R, Baxter WT, Jalife J. Spiral waves of excitation underlie reentrant activity in isolated cardiac muscle. Circ. Research 1993;72:631-650. 23. Lee G, Kumar S, Teh A, Madry A, Spence S, Larobina Goldblatt J, Brown T, Atkinson V, Moten S, Morton JB, Sanders P, Kistler PM, Kalman JM. Epicardial wave mapping in human long-lasting persistent atrial fibrillation: transient rotational circuits, complex wavefronts, and disorganized activity. Eur. Heart J. 2014;35(2):86-97. 24. Ghoraani B, Dalvi R, Gizurarson S, Das M, Ha A, Suszko A, Krishnan A, Chauhan VS. Localized rotational activation in the left atrium during atrial fibrillation: relationship to complex fractionated atrial electrograms and low-voltage zones. Heart Rhythm 2013;10(12): 1830-1838. 25. Pertsov AM, Jalife J. Three-dimensional vortex-like reentry. In: Zipes D, Jalife J, eds. Cardiac Electrophysiology - From Cell to Bedside. Philadelphia: W. B. Saunders; 1995:403-410pp. 26. Yamazaki M, Mironov S, Taravant C, Brec J, Vaquero LM, Bandaru K, Avula UMR, Honjo H, Kodama I, Berenfeld O, Kalifa J. Heterogeneous atrial wall thickness and stretch promote scroll 
waves anchoring during atrial fibrillation. Cardiovasc. Res. 2012; 94: 48-57. 27. Berenfeld O, Pertsov AM. Dynamics of intramural scroll waves in three-dimensional continuous myocardium with rotational anisotropy. J. Theor. Biol. 1999;199: 383-394. 28. Frazier DW, Wolf PD, Wharton M, Tang ASL, Smith WM, Ideker RE. Stimulus-induced critical point. Mechanism for electrical initiation of reentry in normal canine myocardium. J. Clin. Invest.1989;83:1039-1052. 29. Winfree AT. Excitable kinetics and excitable media. In: Marsden JE, Wiggins S, Sirovich L, eds. The Geometry of Biological Time, $2^{\text {nd }}$ edition. Berlin: Springer-Verlag; 2001:258302pp. 30. Atienza F, Almendral J, Jalife J, Zlochiver S, Ploutz-Snyder R, Torrecilla E, Arenal A, J. Kalifa $\mathrm{J}$, Fernández-Avilés F, Berenfeld $\mathrm{O}$. Real-time dominant frequency mapping and ablation of dominantfrequency sites in atrial fibrillation with left-to-right frequency gradients predicts long-term maintenance of sinus rhythm. Heart Rhythm 2008;6(1):33-40. 31. Steinberg JS, Shah Y, Bhatt A, Sichrovsky T, Arshad A, Hansinger E, Musat D. Focal Impulse and rotor modulation: Acute procedural observations and extended clinical follow-up. Heart Rhythm 2017;14:192-197. 32. Cabo C, Pertsov AM, Davidenko JM, Baxter WT, Gray RA. Jalife J. Vortex shedding as a precursor of turbulent activity in cardiac muscle. Biophys. J. 1996;70: 1105-1111. 33. Lee S, Sahadevan J, Khrestian CM, Markowitz A, Waldo AL. Characterization of foci, and breakthrough sites during persistent and long-standing persistent atrial fibrillation in patients: studies using high-density (510-512 electrodes) biatrial epicardial mapping. J. Am. Heart Assoc. 2017;6: e005274. 34. Ahrens CD, Henson R. Hurricanes. In: Meteorology Today: An Introduction to Weather, Climate, and the Environment, $12^{\text {th }}$ edition. Boston: Cengage; 2019:439-471 pp. 35. Chernov AA. Formation of crystals in solutions. Contemp. Phys. 1989;30:251-276.

Permission Letter for Figure 4B of Ghoraani that is used in Figure 1D

\section{Supplemental Materials}

Figure 3 Data and weblink

Figure 4 Data and weblink

Figure 7 Data and weblink

Figure 8A Data and weblink

Figure 8B Data

Figure 9 Data and weblink

\section{TABLES}

\begin{tabular}{llllllllllll}
\hline Blue & $\mathrm{e}_{1}$ & & & Red & $\mathrm{e}_{2}$ & & & Skew & $\mathrm{e}_{2}$ & \\
\hline $\mathrm{x}$ & $? \mathrm{x}$ & $?$ & Deg & $\mathrm{x}$ & $? \mathrm{x}$ & $?$ & $\mathrm{Deg}$ & $\mathrm{x}$ & $? \mathrm{x}$ & $?$ & $\mathrm{Deg}$ \\
3.54 & & & 15.7 & 3.46 & & & -16.2 & 3.47 & & & -9.3 \\
2.56 & 0.98 & 10.2 & 21.3 & 2.44 & 1.02 & 9.8 & -22.3 & 2.47 & 1.01 & 10 & -11.9 \\
1.59 & 0.97 & 10.3 & 32.2 & 1.40 & 1.04 & 9.6 & -35.5 & 1.45 & 1.01 & 9.8 & -16.6 \\
0.66 & 0.93 & 10.7 & 56.6 & 0.30 & 1.11 & 9.1 & -73.5 & 0.42 & 1.03 & 9.7 & -27.2 \\
-0.22 & 0.88 & 11.5 & 192.2 & -0.86 & 1.16 & 8.6 & -130.8 & -0.68 & 1.11 & 9.1 & -66.7 \\
-1.12 & 0.9 & 11.1 & 138.1 & -1.92 & 1.06 & 9.4 & -152.5 & -1.89 & 1.20 & 8.3 & -140.6 \\
-2.07 & 0.95 & 10.5 & 154.2 & -2.95 & 1.02 & 9.8 & -161.3 & -2.94 & 1.05 & 9.5 & -159.3 \\
-3.05 & 0.98 & 10.2 & 161.9 & -3.96 & 1.01 & 9.9 & -165.8 & -3.96 & 1.02 & 9.8 & -166.1 \\
\hline
\end{tabular}

Table I. WF strikes positions at $\mathrm{e}_{1}, \mathrm{e}_{2}$ and skewed $\mathrm{e}_{2}$ along the $\mathrm{v}_{\mathrm{s}}$ vector path.

Values of $x$ represent the distance $S_{R}$ is from point $p$ in Figure 3. Note that as $S_{R}$ approaches the inflection point, there is an increasing frequency (?) on strong-side (blue), while simultaneous decreasing ? on weakside (red).

\section{FIGURE LEGENDS}


Figure 1. Three components of electrode recording pattern of a migrating rotor breaching perimeter of electrodes.

1. Unipolar recordings of a clockwise spinning rotor as it migrates from within ring of 16 electrodes. (Methods in (12)). A head-meets-tail activation sequence (slanted left dotted yellow oval) is observed as $\mathrm{CW}$ rotor spins within a circular perimeter of electrodes. As rotor migrates between electrodes $\mathrm{e}_{10}$ and $\mathrm{e}_{11}$, three events occur: simultaneous diametric frequency shifts (strong-side (s) $\mathrm{e}_{10}$ increases in frequency while weak-side (w) decreases in frequency), an unpaired WF strike occurs on strong-side $\mathrm{e}_{10}$, and a reversal of activation sequence $\mathrm{e}_{8}-\mathrm{e}_{13}$ (yellow arrows).

2. Schematic of rotor positions of A above, as core migrations breaches perimeter of electrodes. Actual recordings (12) included unipolar, adjacent bipolar and cross-circle bipolar confirming the breach.

3. Schematic timing of WF strikes at $\mathrm{e}_{8}-\mathrm{e}_{13}$. Note the 3-component pattern of WF strikes that occurs when a rotor breaches the perimeter; simultaneous strong-side increasing frequency with a weak-side decreasing frequency, an unpaired strong-side WF strike, and a reversal of activation sequence. This pattern was observed repeatedly 54 of 56 transits (12).

4. Independent confirmation of the 3-component pattern of diametric property is seen in recordings of a counterclockwise spinning rotor meandering across a circular array of electrodes. Strong-side (s) and weak-side (w) have been labelled here, showing an unpaired strong-side WF strike, and reversal of activation sequence (green dashed oval with arrow). Reprinted and adapted Fig 4B from Ghoraani et al (24) with permission from Elsevier Publishing Copyright 2013, Heart Rhythm.

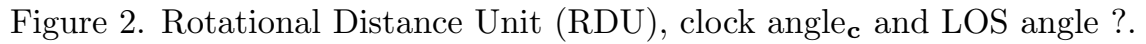

A. The $\mathrm{S}_{\mathrm{R}}$ is theoretical clock that slides on a surface at a constant velocity. The clock hand represents a

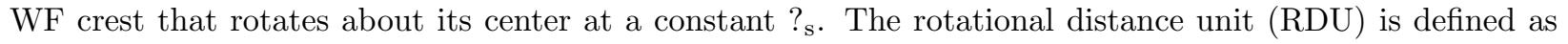
a single revolution/distance travelled 2?/D. Regions of WF that exhibit greatest velocity, shear zones, and regions of slowest velocity, stall zones are separated by consecutive RDUs.

B. Top : The rotational WF of an $\mathrm{S}_{\mathrm{R}}$ is represented as a green clock hand that spins clockwise at constant $?_{\mathrm{s}}$. Recording electrodes $\mathrm{e}_{1}$ and $\mathrm{e}_{2}$ are on the tissue surface. $\mathrm{S}_{\mathrm{R}}$ moves at a constant $?_{\mathrm{s}}$ along its vector path (green arrow) that is a perpendicular bisector between $e_{1}$ and $e_{2}$, such that the rotor is always equidistant to $e_{1}$ and $e_{2}$. The distance travelled between each complete revolution is the RDU.

Bottom: Each RDU (vertical dotted lines) is shown as the clock approaches inflection point (p ). The colored dots correspond to the time at which the wave front strikes the electrodes. The clockwise rotation in the plan of atrial surface results in sequential activation of $e_{1}$ followed by $e_{2}$. With each RDU, the time period between WF strikes at e1 shortens, while time between WF strikes at $\mathrm{e}_{2}$ lengthens. When rotor passes inflection point $\mathbf{p}$, the sequence reverses. Changing the clock rotation to counterclockwise would reverse the sequence changes.

C. (a). Clock Angle $\psi_{c}$. Upper inset shows angle $0^{\circ}$ is set at forward vector path of motion at the 3:00 position. During CW spin, the clock hand (thin yellow triangle) of the source ( $\mathbf{S}$ ), is initially positioned at 9:00 $(\pi)$. The source's clock hand rotates with constant? $?_{\mathbf{s}}$ at $10 \mathrm{~Hz}(20 \pi / \mathrm{s})$. The clock hand is a positive angle at $\pi$ and decreases as $\psi_{c}$ rotates towards 3:00 passing $+\pi / 2$ set at 12:00. When clock hand passes 12:00, fastest forward tangential velocity is parallel to? ${ }_{\mathbf{s}}$ that may create a potential WF shear zone at greater radii. Once the clock hand passes 3:00 (0 radians), $\psi_{S}$ is a negative angle as it rotates back towards 9:00 ( $\left.-\pi\right)$, passing $-\pi / 2$ set at $6: 00$. As the clock hand passes $6: 00$, the slowest forward velocity is parallel to ? that may create a potential WF stall zone at greater radii. The RDU (small clocks along vector ?s ) is shown as the clock hand makes one complete revolution. Shear and stall regions would be expected to occur with each revolution at same respective $\psi_{c}$, separated by distance D of the RDU. Electrodes $e_{1}$ and $e_{2}$, depicted at the center of synchronized clocks, are positioned $1 \mathrm{~cm}$ on either side of the vector path that is a perpendicular bisector. Four subsequent WF strikes are shown at each electrode as $\mathbf{S}$ moves passed the inflection point $\mathbf{p}$.

D. Line of Sight (LOS) angle ?. Note that a WF strike occurs when $\psi_{c}=$ LOS angle ? as it sweeps past clock with each rotation. Upper inset shows that angle $0^{\circ}$ is set directly along the forward vector path of 
the source. The positive and negative angle ?s are set to align with the positive and negative angle $\psi_{\mathrm{c}}$ as seen in 3C. Note that onceS passes the inflection point $\mathbf{p}$, LOS angle ? is obtuse. The change in angle ? (??) between subsequent WF strikes is a positive value at a strong-side electrode, and negative value at a weak-side electrode. Right inset shows sequence of WF strikes is $\mathrm{e}_{1}-\mathrm{e}_{\mathrm{v}}-\mathrm{e}_{2}$. Since $\mathrm{e}_{1}$ precedes $\mathrm{e}_{\mathrm{v}}$ (dashed white circle), then $\mathrm{e}_{1}$ sits on the strong-side.

Figure 3. Electrodes on either side of $\mathrm{S}_{\mathrm{R}}$ 's vector path reveals the diametric property of RFE.

A. Source's clock, and electrode clocks are synchronized, aligned, and coplanar. Clockwise $\mathrm{S}_{\mathrm{R}}$ with constant angular velocity $20 \pi / \mathrm{sec}$, moves at constant velocity $10 \mathrm{~cm} / \mathrm{sec}(\mathrm{RDU}=2 \pi / 1 \mathrm{~cm})$ along a perpendicular bisector vector path of line $\mathrm{e}_{1} \mathrm{e}_{2}(2.0 \mathrm{~cm}$ apart $)$. Electrodes $\mathrm{e}_{1}$ and $\mathrm{e}_{2}$ are equidistant to $\mathrm{S}_{\mathrm{R}}$ along its entire path. Clocks at $\mathrm{e}_{1}$ and $\mathrm{e}_{2}$ constructed with each radius $1.0 \mathrm{~cm}$ touch each other at the common tangent point p. The source clock reads 9:00 when the source is $3 \mathrm{~mm}$ away from pointp. One complete revolution of the clock occurs every $1 \mathrm{~cm}(\mathrm{RDU}=2 \pi / 1 \mathrm{~cm})$ as the source moves toward the electrodes. The clock hand (yellow triangle) extends beyond the perimeter of the clock, rotates and strikes $\mathrm{e}_{1}$ before striking $\mathrm{e}_{2}$. The first 3 RDUs result in WF strikes at $e_{1}$ at line $a, b$, and $c$, and at $e_{2}$ at lines $d$, e, and $f$. The distance fromp and the angle of the WF strikes are listed in table 1 . The clock hand strikes the $\mathrm{e}_{1}$ clock earlier in its rotation (strong-side) while striking $e_{2}$ later in its rotation (weak-side). See Supplementary Materials Figure 3 Data File. B. Graphical presentation of both $\mathrm{f}(\mathrm{x})=\psi_{\mathrm{c}}$ for clock hand angle and $\mathrm{f}(\mathrm{x})=$ ? for LOS angle to both electrodes as $\mathrm{S}_{\mathrm{R}}$ approaches and recedes from inflection point $\mathbf{p}$, the closest approach to electrodes. Note $\mathrm{x}$-axis is reversed. Angle ? from the source to $\mathrm{e}_{1}$ and $\mathrm{e}_{2}$ is calculated by the arccotangent for strong-side (blue curve) and the arccotan curve for weak-side (red curve) from Equation 13 and Equation 14 respectively. Starting point for first RDU (black dot with dashed arrow), begins with the source clock hand at 9:00 ( $\pi$ radians) at a position $3 \mathrm{~cm}$ away from point $\mathbf{p}$. The RDU lines of the clock angle $\psi_{\mathrm{c}}$ (green lines) intersect the arccot curves for the LOS ? at the moment of WF strike at $e_{1}$ and $e_{2}$ (white circles). Intersection points at a-f correspond to the distance from $\mathbf{p}$ and clock angles shown in $3 \mathrm{~A}$ above and listed in Table 1 . RDUs \#1-3 show WF strikes at a-c at $e_{1}$ and d-f at $e_{2}$. Arccot curves are shown for comparison if $e_{2}$ sits closer to the inflection point at distances $\frac{1}{2}, \frac{1}{4}$, and $1 / 8$ of $\mathrm{r}_{2}$ (black curves). C. Similar to A, the $\mathrm{S}_{\mathrm{R}}$ clock, and electrode clocks are all synchronized, aligned, and coplanar. The vector path of the source is at a tilted $(=$ $30^{\circ}$ ) compared to $\mathrm{A}$. The path is created such that $\mathrm{e}_{1}$ is still $1 \mathrm{~cm}$ from the vector's path of closest approach. The inset of the enlarged electrodes shows the trigonometry used to calculate the transverse common tangent line segment length (tct) and the $\mathrm{e}_{2}$ distance to the closest approach of the source $\left(\mathrm{r}_{2}\right)$. Electrodes $\mathrm{e}_{1}$ and $\mathrm{e}_{2}$ remain at their same position with $\mathrm{L}=2.0 \mathrm{~cm}$ of separation. The tct is $1.0 \mathrm{~cm}$ (tct $=\mathrm{L}$ sin ). The distance $\left(\mathrm{r}_{2}\right)$ of $\mathrm{e}_{2}$ to the point of closest approach of the $\mathrm{S}_{\mathrm{R}}$ 's vector path is calculated at $0.73 \mathrm{~cm}\left(\mathrm{r}_{2}=\mathrm{x}_{2} \cos \right)$. D. Similar to B, simultaneous plots of the clock hand angle $\psi_{\mathrm{c}}$ and LOS angle ? as function of distance from both electrodes. The plot of $\psi_{c}$ and the arccot curve for ? of the LOS to $e_{1}$ remains the same. The arccot curve for $\mathrm{e}_{2}$ is calculated for an $\mathrm{r}_{2}=0.73 \mathrm{~cm}$ and skewed by the tct $=1.0 \mathrm{~cm}$. Intersection of curves identify when and where WF strikes occur. RDUs \#1-3 show WF strikes at a-c at $\mathrm{e}_{1}$ and d-f at $\mathrm{e}_{2}$.

Figure 4. The diametric property of an $\mathrm{S}_{\mathrm{R}}$ at different distances of equidistant electrodes.

$\mathrm{S}_{\mathrm{R}}$ has a $?_{s}=5 \mathrm{~Hz}$, that moves with $?_{\mathrm{s}}=1 \mathrm{~cm} / \mathrm{s}$ along a vector path is a perpendicular bisector between $\mathrm{e}_{1}$ and $\mathrm{e}_{2}$. Electrode pairs sit at $1 \mathrm{~mm}, 2 \mathrm{~mm}, 5 \mathrm{~mm}, 1 \mathrm{~cm}$ and $2 \mathrm{~cm}$ from the inflection point p. Computed WF strikes are plotted as a function of $\mathrm{x}$ from the $\mathrm{p}$ with the instantaneous frequency (hollow circles) on the y-axis. RDU is $2 ? / 0.2 \mathrm{~cm}$. Frequency effects on the strong-side and weak-side of the $\mathrm{S}_{\mathrm{R}}$ are shown by smoothed blue shaded curves and red/yellow curves respectively for a particular electrode distance. The approaching $\mathrm{S}_{\mathrm{R}}$ shows that the sequence of WF strikes occur at $\mathrm{e}_{1}$ immediately precedes $\mathrm{e}_{2}$. After $\mathrm{S}_{\mathrm{R}}$ passes inflection $\mathrm{p}($ at $\mathrm{x}=0)$, the sequence of WF strikes reverses, $\mathrm{e}_{2}$ strikes precede $\mathrm{e}_{1}$ (hollow black ovals). At about $0.5 \mathrm{~cm}$, marked separation of progressive WF strike frequency occurs comparing strong and weak-sides (blue and red shaded ovals). At far left and right of the plot, frequency approaches $?_{s}$ at $5 \mathrm{~Hz}$. An unpaired WF strike on the strong-side (black bracket) occurs at the inflection point for each distance. Closer electrodes show more abrupt and greater frequency changes than electrodes that straddle the vector path at further distances. Equations and data from these plots are provide in the Supplementary Materials Figure 4 Data 
File.

Figure 5. Archimedean spiral and time reference.

A source $\mathrm{S}_{\mathrm{Sp}}$ emits a clockwise Archimedean spiral WF governed by equation $\rho=\psi$ sp for 2 full rotations with a constant wavelength $?=2$ ?

Expanded view of first revolution of spiral with defining position of clock hand ( $\psi_{\mathrm{c}}$ grey triangle) along the first ?. The length of line from 0 to 2 ? is the spiral wavelength ? that defines its size. Radial spokes from center to the spiral arm show the spiral angles $\psi_{\mathrm{Sp}}$ at increments of $30^{\circ}$, or ?/6 radians, and increases in the CCW direction. The clock hand angle $\psi_{\mathrm{c}}$ is measured by having the $0^{\circ}$ angle aligned at 3:00, the vector path of $\psi_{\mathrm{Sp}}$. An example point of reference, the spiral angle $\psi_{\mathbf{S p}}=90^{\circ}$ is noted at the arrow.

C. As spiral source $\mathrm{S}_{\mathrm{Sp}}$ spins clockwise ?/4 radians, a point on the spiral wave can still be computed by a spiral summation angle $\psi_{\mathrm{SA}}$. The $\psi_{\mathrm{SA}}$ is generated by first identifying the clock hand angle $\psi_{\mathrm{c}}$ compared to the zero angle and then following the wave back CCW by the spiral angle $\psi \mathbf{s p}$. The clock angle has positive values above the line $\mathrm{v}_{\mathrm{s}}$ and negative values below line $\mathrm{v}_{\mathrm{s}}$. The new position of spiral angle $\psi \mathbf{s p}$ $=90^{\circ}$ is noted at the arrow. D. The rotational distance unit (RDU, 2?/D) is applied to spiral wave. The distance $\mathrm{D}$ travelled is function a constant velocity $?_{\mathrm{s}}$ of the spiral as it makes one completes revolution at a constant angular velocity, ? . The 3-point sequence rule developed for rotational waves is used here to label the strong-side and weak-sides of a spiral wave (blue and red rectangles, respectively). Similar to Fig 3(a), separated by D distances, WFs would move faster through strong-side shear zones and slower through weak-side move slower (stall zones).

Figure 6. Spiral math components determine WF strikes.

$\mathrm{A}$ and B. A moment in time that a clockwise spinning $S_{S p}$ (green striped curve) approaches the inflection point (p) between 2 equidistant electrodes $\mathrm{e}_{1}$ and $\mathrm{e}_{2}\left(\mathrm{r}_{1}=\mathrm{r}_{2}\right.$, center of blue and red circles) on either side of the vector path $\mathbf{v}_{\mathbf{s}}$ of $\mathrm{S}_{\mathrm{Sp}}$. Step 1: determine the clock hand angle (thin gray triangle aligned along ray from $0->2$ ?, or spiral's $1^{\text {st }}$ ?) as measured from the $0^{\circ}$ angle represented by the forward vector path $\left(\mathbf{v}_{\mathbf{s}}\right)$ of $\mathrm{S}_{\mathrm{Sp}}$ (same as in Fig 3A). Step 2: determine the distance from $\mathrm{S}$ to $\mathrm{e}_{1}$, the hypotenuse $\mathrm{h}$, of the right triangle $\mathrm{S}-\mathrm{p}-\mathrm{e}_{1}$. The distance $h$ is converted to the angle $\psi_{\eta}$ and is plotted on the spiral curve in a counterclockwise direction from $\psi_{\mathrm{c}}$. The resultant point on the spiral, at the $\psi_{\Sigma A}$, in 2-D space identifies that the spiral WF crest did not intersect with $\mathrm{e}_{1}$ at that position and time. C and D. Two examples show when the spiral WF crest strikes $e_{1}$. When $S_{S p}$ is at position $\mathbf{S}_{\mathbf{S p 1}}$ (approaching the inflection pointp) and at position $\mathbf{S}_{\mathbf{S p} \mathbf{2}}$ (receding from point $\mathbf{p}$ ). At $\mathbf{S}_{\mathbf{S p} \mathbf{1}}$, the clock hand angle $\psi_{\mathrm{c} 1}$ is added to the hypotenuse angle $\psi_{\eta 1}$ resulting in an acute angle $\psi_{\Sigma A}$ that is equal the acute LOS angle $?_{1}$. At $\mathbf{S}_{\mathbf{S p} 2}$, the clock hand angle $\psi_{\mathrm{c} 2}$ is added to the hypotenuse angle $\psi_{\eta_{2}}$ resulting in an obtuse angle $\psi_{\Sigma A}$ that is equal the obtuse LOS angle ? $\mathbf{2}_{\mathbf{2}}$. Close inspection of $\mathbf{S}_{\mathbf{S p} \mathbf{1}}$ shows that the WF strike at $\mathrm{e}_{\mathbf{1}}$ has occurred before a WF strike would occur at $e_{2}$, whereas the sequence is reversed when source is passed $\mathbf{p}$ at position $\mathbf{S}_{\mathbf{S p 2}}$. Here, the WF strike at $\mathrm{e}_{2}$ had already occurred just before the strike at $\mathrm{e}_{1}$.

Figure 7. Equidistant electrodes on either side of source's vector path reveals the diametric property of spiral WF wave frequency effects.

A. $\mathrm{S}_{\mathrm{Sp}}$ has a wavelength of ? radians, a spiral size ? = ? cm and spins with an angular velocity of 10 rotations $/ \mathrm{s}(20 ? / \mathrm{s})$. $\mathrm{S}_{\mathrm{Sp}}$ moves on a vector path at $v_{s}=15 \mathrm{~cm} / \mathrm{s}$ that is a perpendicular bisector between electrodes $\mathrm{e}_{1}$ and $\mathrm{e}_{2}$, each $0.5 \mathrm{~cm}$ from the point of closest approach (transverse common tangent to blue and red circles). A reference position of $\mathrm{S}_{\mathrm{Sp}}$ at $3 \mathrm{~cm}$ (thick green spiral) from the inflection point between electrodes is the spiral source's clock angle ${ }_{\mathrm{c}}$ is at 9:00 or ? radians. The spiral wave in its $\mathrm{x}$ position and spin orientation is shown at each WF strike as calculated to occur at positions 1-13. WF strikes at $e_{1}$ (bold dash-dot spirals) are compared to WF strikes at $e_{2}$ (dashed spirals). Black and yellow dashed spirals show the sequence reversal of WF strikes secondary to the diametric property by opposite and progressive changes of T comparing strong and weak-sides (positions 7-9). Strong-side has one more WF strike than weak-side (unpaired WF strike at position 9), identical to that seen during rotor migration breaching perimeter of 
electrodes (2) that we described as a $\frac{1}{2}$ cycle drop-off. See Supplementary Materials Figure 7 Data File. B. The LOS angle? is plotted as a function of distance $\mathrm{x}$ to inflection point by the arccotangent curves for electrodes $e_{1}$ and $e_{2}$ (blue and red curve, respectively). Superimposed on graph is the spiral clock angle $\psi_{\mathrm{c}}$ (green lines), the distance to electrodes in radians as the hypotenuse angle $\mathrm{h}$ (small portion of the pink parabolic curve is illustrated), the spiral summation angle sA (black lines). The points of intersection of SA with the arccotangent curves (white circles) indicate when $\mathrm{SA}^{=}$?, identifying a WF strike. Although both $e_{1}$ and $e_{2}$ record faster frequencies during an approaching $\mathrm{S}_{\mathrm{Sp}}$ compared to receding, the WF strikes at $\mathrm{e}_{1}$ during the approach accelerate $\left(\mathrm{S}_{\mathrm{Sp}}\right.$ positions at $\left.1,3,5,7\right)$ while simultaneously the WF strikes at $\mathrm{e}_{2}\left(\mathrm{~S}_{\mathrm{Sp}}\right.$ positions at $2,4,6,8)$ decelerate. Example for frequency determination at WF strikes at $\mathrm{e}_{1}$ from position 5 to position 7 can be calculated by either ?ps ${ }_{\mathrm{c}}$ or ? $\mathrm{x}$. The ?ps $\mathrm{c}_{\mathrm{c}}$ method would be via using $\mathrm{ps}_{\mathrm{c}}$ values at hollow stars $(-2.941,-0.779$, respectively) and plugging into frequency equation. 35 , or by the ?x method by equation 36. C. Side-Dependent Frequency Changes of Spiral Wave. Plots of the instantaneous frequencies at each WF strike are shown for both strong and weak electrodes ( $e_{1}$ and $e_{2}$, respectively). The approaching spiral wave shows a progressive increase in frequency of WF strikes at the strong-side electrode $e_{1}$. Simultaneously, there is a progressive decrease in frequency of WF strikes at the weak-side electrode $e_{2}$. This diametric progression leads to an unpaired $\mathrm{WF}$ at $\mathrm{e}_{1}$ (position 9, near the inflection point, $\mathrm{p}$ ) resulting in the sequence reversal with WF strikes at $e_{2}$ preceding $e_{1}$. From relatively far distances $(x>>r)$ during the approach, strong and weak-sides frequencies of WF strikes are nearly the same, at $14.78 \mathrm{WF}$ strikes /sec. When $\mathrm{S}_{\mathrm{Sp}}$ recedes from $\mathrm{p}$, frequencies of both strong and weak-sides then approach a final receding frequency of spiral $5.22 \mathrm{WF}$ strikes/sec as per Equation 40 or $10 \pm 4.78 \mathrm{WF}$ strikes/sec.

Figure 8. Comparison of frequencies observed from a moving spinning $\mathrm{S}_{\mathrm{Sp}}$ source and a moving $S_{C}$.

A. Comparison of frequency changes with propagation of a spiral wave between different distances of equidistant electrodes. The speed of spiral source $\mathrm{v}_{\mathrm{s}}=1 \mathrm{~cm} / \mathrm{sec}$. Angular frequency is 5 rotations $/ \mathrm{sec}, \mathrm{RDU}=$ $0.2 \mathrm{~cm}^{-1}$, with $?=? \mathrm{~cm}$. Comparison of calculations were made with stationary electrodes placed at $0.2 \mathrm{~cm}$, $03 \mathrm{~cm}, 0.5 \mathrm{~cm}$, and $2 \mathrm{~cm}$ from the inflection point p. Closer electrodes to the $\mathrm{S}_{\mathrm{Sp}}$ center create a greater diametric change in ? between the strong and weak-side electrodes (gradation of darker blue and darker red lines, respectively). Similar to $\mathrm{CDE}, \mathrm{S}_{\mathrm{Sp}}$ at larger distances from electrodes, the approaching ? is faster than $?_{s}$, then gradually decreases, becoming less than $?_{s}$ after receding from $\mathrm{p}$ (light blue and light yellow lines, respectively). Using Equation 40, the approaching $?=5.32 \mathrm{~Hz}$ and the receding $?=4.68 \mathrm{~Hz}$. See Supplementary Materials Figure 8A Data File. B. Classical Doppler effect on ? changes sensed by equidistant electrodes (same as in (a) above) of a moving source $\mathrm{S}_{\mathrm{c}}$ (same $?_{s}=1 \mathrm{~cm} / \mathrm{s}$ ) that emits waves propagating centrifugally away at ? $\mathrm{cm} / \mathrm{s}$ (same as spinning spiral source above if not moving in $\mathrm{x}$-direction) utilizing Equation 21. For this to occur, with same ? at ? $\mathrm{cm}$ and same frequency at $5 \mathrm{~Hz}$, the wave propagation velocity is $5 ? \mathrm{~cm} / \mathrm{s}$. If the electrode was directly on the $?_{s}$ vector path, the approaching $?=5.34 \mathrm{~Hz}$, and the receding $?=4.70 \mathrm{~Hz}$ ). Note that from far away, greater electrode distance, the WF strike $?$ is almost identical to that of a spiral wave at a distance (several ? away). See Supplementary Materials Figure 8B Data File.

Figure 9. Spiral size on diametric property of SFE.

Plots of different spiral sizes vs frequency are shown when electrodes $e_{1}$ and $e_{2}$ (each $2 \mathrm{~mm}$ from $\mathbf{p}$ ) are equidistant to $S_{S p}$ along its vector path of motion. $S_{S p}$ moves along the vector with constant $?_{\mathrm{s}}=1 \mathrm{~cm} / \mathrm{sec}$, while spinning at constant $?_{s}=5 \mathrm{~Hz}$, with $\mathrm{RDU}=2 ? / 0.2 \mathrm{~cm}$. Curves are smoothed lines linking the subsequent WF strikes of $S_{S p}$ of same size ?. Pairs of curves show frequency differences between strong-side $\mathrm{e}_{1}$ (light or dark green dots) and weak-side $\mathrm{e}_{2}$ (light or dark pink dots). At far distances $(\mathrm{x}>>\mathrm{r})$, either approaching or receding, the WF strikes at $e_{1}$ and $e_{2}$ from the largest spiral size $(200 ? \mathrm{~cm})$ have values near $?_{s}$. As ? gets smaller, the approaching ? is faster, and receding ? is slower. The greatest side-dependent difference occurs near the inflection point p. An unpaired WF strike on the strong-side (3 dark blue arrows) with that on the weak-side (2 red arrows) results in the final step before sequence reversal is complete (dashed ovals). When ? = D at $0.2 \mathrm{~cm}$, the approaching $\mathrm{WF} ?$ is $10 \mathrm{~Hz}$ (twice the value of $?_{s}$ ), but when receding 
from p, no WF strides occur (electrodes would be surfing between spiral WFs). See Supplementary Materials Figure 9 Data. Figure 10. The side-relative differences of frequency observed from $S_{R}$ or $S_{S p}$ are a result of the diametric property of RFE and SFE seen at all levels of nature. A. Person R moves along a path between persons $\mathrm{S}$ and $\mathrm{W}$ while spinning $1 / 2$ turn. Person $\mathrm{W}$ observed 0 turns since person $\mathrm{R}$ faced person $\mathrm{W}$ along its entire path. Person S observed 1 turn. B. Person R moves along a path between persons S and $\mathrm{W}$ with a faster spin, completing 1 and $1 / 2$ turns over the same distance. Person S observed 2 turns. Person $\mathrm{W}$ observed 1 turn. C. A rotating binary star exhibits peak Doppler effect frequencies when the tangential vector path of the small orange star is directed toward observers and the location of the peak frequency can be considered as the clock angle ${ }_{c}$ for an $S_{R}$. When binary star moves on path between $\mathrm{S}$ and $\mathrm{W}$, the timing of peaks will exhibit diverging trends of the diametric property of $S_{R}$. Yet, for observers $D$ and D' that travel with the binary star, stationary in the same inertial plane, then they perceive no diverging frequency shifts and no difference in count of peak frequencies. The side-dependent timing of peak frequencies exhibit the diametric property of RFE that is relative to motion. On a grander, and slower scale, if one were to replace the binary with a star within a spinning and moving spiral galaxy, then the peak frequency count and timing changes between peaks must similarly follow the diametric property of SFE.

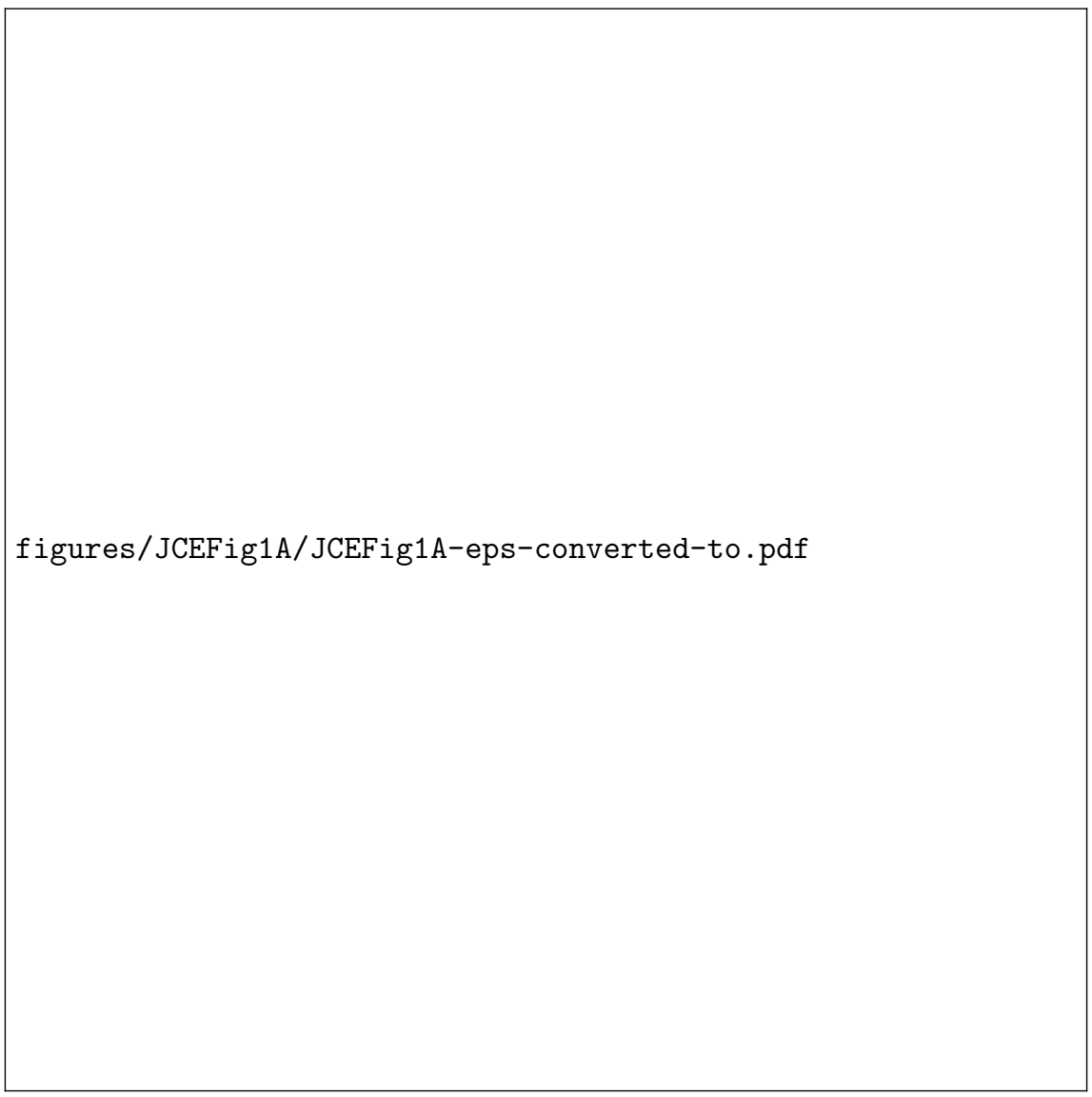


figures/JCEFig1BC/JCEFig1BC-eps-converted-to.pdf 
figures/JCEFig1D/JCEFig1D-eps-converted-to.pdf 
figures/JCEFig2AB/JCEFig2AB-eps-converted-to.pdf 
figures/JCEFig2C/JCEFig2C-eps-converted-to.pdf 
figures/JCEFig2D/JCEFig2D-eps-converted-to.pdf 
figures/JCEFig3ABCDeps/JCEFig3ABCDeps-eps-converted-to.pdf 
figures/JCEFig4/JCEFig4-eps-converted-to.pdf 
figures/JCEFig5/JCEFig5-eps-converted-to.pdf 
figures/JCEFig6/JCEFig6-eps-converted-to.pdf 
figures/JCEFig7AB/JCEFig7AB-eps-converted-to.pdf 
figures/JCEFig7C/JCEFig7C-eps-converted-to.pdf 
figures/JCEFig8A/JCEFig8A-eps-converted-to.pdf 
figures/JCEFig8B/JCEFig8B-eps-converted-to.pdf 
figures/JCEFig9/JCEFig9-eps-converted-to.pdf 
figures/JCEFig10ABC/JCEFig10ABC-eps-converted-to.pdf 